Document downloaded from:

http://hdl.handle.net/10251/79515

This paper must be cited as:

Chen, Y.; Liu, T.; García Gil, PJ.; Albertos Pérez, P. (2016). Analytical design of a generalised predictor-based control scheme for low-order integrating and unstable systems with long time delay. IET Control Theory and Applications. 10(8):884-893. doi:10.1049/ietcta.2015.0670.

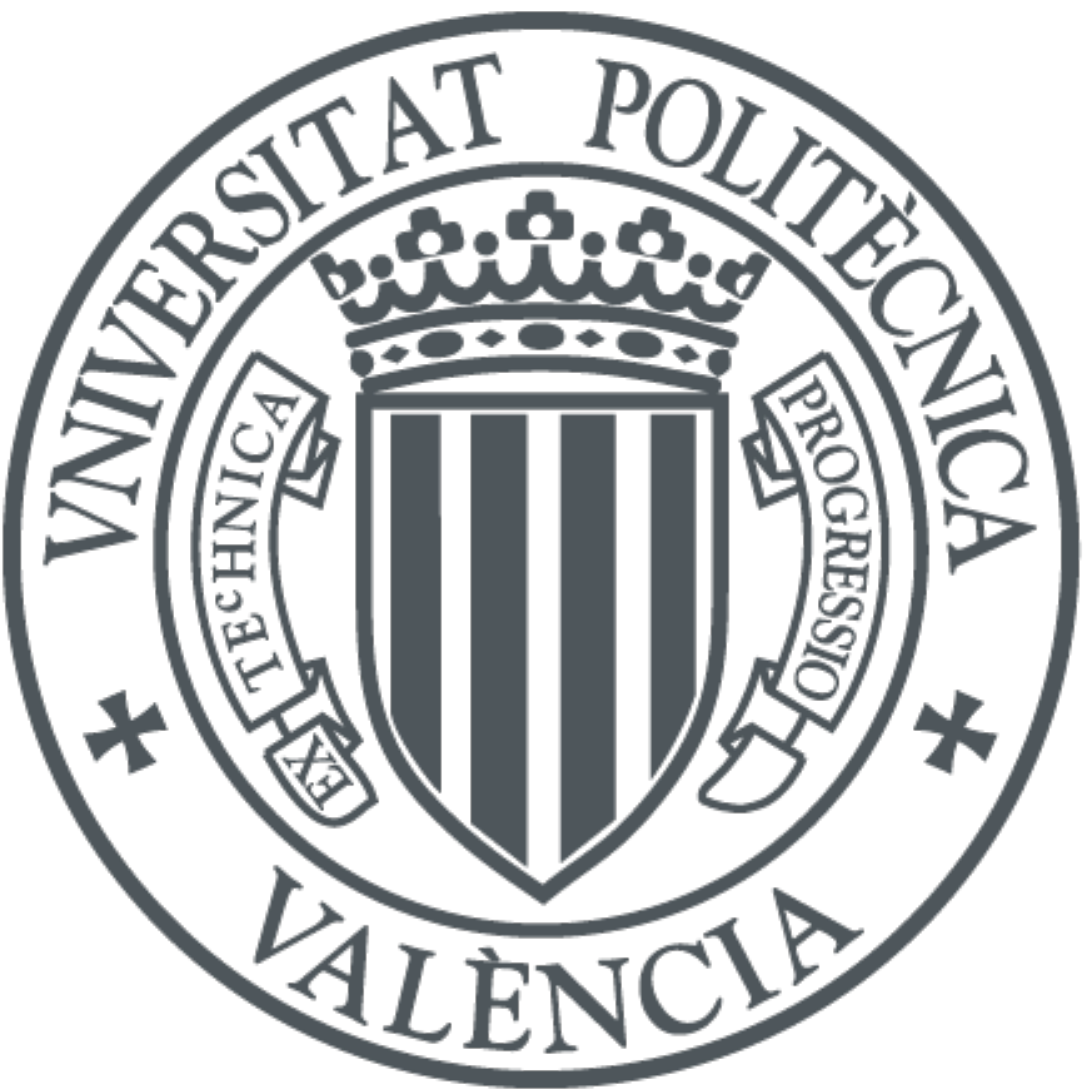

The final publication is available at

http://dx.doi.org/10.1049/iet-cta.2015.0670

Copyright Institution of Engineering and Technology (IET)

Additional Information 


\title{
Analytical design of a generalized predictor-based control scheme for integrating and unstable systems with long time delay
}

\author{
Yueling Chen ${ }^{a}$, Tao Liu ${ }^{a}{ }^{*}$, Pedro García ${ }^{b}$, Pedro Albertos ${ }^{b}$ \\ a Institute of Advanced Control Technology, Dalian University of Technology, Dalian, 116024, P. R. China \\ b Instituto Universitario de Automática e Informática Industrial (AII) Universidad Politécnica de Valencia, Spain \\ * Corresponding author. Tel: +86-411-84706465; Fax: +86-411-84706706 \\ E-mail addresses: liurouter@ieee.org (T.Liu), Y.L.Chen.dlut@gmail.com (Y. Chen), \\ pggil@isa.upv.es(P.García),pedro@aii.upv.es (P.Albertos)
}

\begin{abstract}
In this paper, the problem of controlling integrating and unstable systems with long time delay is analyzed. Based on a generalized predictor-based control structure, where the plant time delay can be taken out of the control loop for the nominal plant, an analytical design of the controllers is proposed in terms of the delay-free part of the nominal plant model. Correspondingly, further improve control performance is obtained compared with recently developed predictor-based control methods relying on numerical computation for controller parameterization. The load disturbance rejection controller is derived by proposing the desired closed-loop transfer function, and another one for set-point tracking is designed in terms of the $\mathrm{H}_{2}$ optimal control performance specification. Both controllers can be tuned relatively independently in a monotonic manner, with a single adjustable parameter in each controller. The tuning procedure allows to realize the best trade-off between the control performance and robustness against process uncertainties. By establishing the sufficient and necessary condition for holding robust stability of the closed-loop control system, tuning constraints are derived together with numerical tuning guidelines for the disturbance rejection controller. Illustrative examples taken from the literature are used to demonstrate the effectiveness and merit of the proposed method.
\end{abstract}

Keywords: Time delay system; discrete-time model; two-degree-of freedom (2DOF) control; output prediction; robust stability 


\section{Introduction}

It has been widely recognized that integrating and unstable processes are difficult to control by using the conventional unity feedback control structure, especially under the presence of time delay. Time delay is usually associated with industrial process operations, due to mass transportation, energy exchange, and signal processing etc ${ }^{1,2}$. For example, the heating-up process of an industrial injection molding machine is a typical integrating process with time delay, since the temperature of the heating barrel will not be changed after the electric heater is turned on for a certain time due to the slow exchange of massive thermal energy, and then rise up continuously rather than reaching a steady value. In the recent years a lot of efforts have therefore been devoted to develop advanced control methods for these processes. Concerning the use of a proportional-integral (PI) or proportional-integral-derivative (PID) controller, improved tuning methods were proposed in the references ${ }^{3-8}$ to enhance disturbance rejection performance. Due to the fact that the standard internal model control (IMC) structure cannot hold internal stability for integrating and unstable processes ${ }^{9}$, a few IMC-based control schemes were developed in terms of a two-degree-of-freedom (2DOF) control strategy in the literature ${ }^{10-16}$, based on using different tracking error specifications and stability margins. In dealing with time delays, the Smith predictor (SP) has been effectively used to control stable processes with long time delay ${ }^{17}$, but it cannot be used for integrating and unstable processes due to the problem of internal instability ${ }^{18}$. A few modified SP control methods have been recently developed to improve control performance for integrating and unstable processes ${ }^{19-34}$. In the recent papers ${ }^{35,36}$, a generalized predictor (GP) structure was developed to control stable, integrating or unstable processes with long time delay. The proposed GP is equivalent to the SP but can be applied to integrating and/or unstable systems. Moreover, the delay is eliminated from the characteristic equation of the closed-loop system transfer function. However, no specific performance specifications, controller tuning methodology, or stability constraints were studied therein for engineering applications.

In this paper, the GP-based structure given in the paper $^{36}$ is studied for integrating and unstable systems with long time delay, reducing the control design problem to that for non-delayed plants in terms of the nominal case that the time delay can be taken out of the control 
loop, but taking into account the uncertainties in the original delayed plant. Explicit controller design formulae are derived based on using a discrete-time plant model with time delay and the zero/pole distribution in the z-plane. As a result, both the set-point tracking response and the load disturbance rejection response can be regulated relatively independently through the corresponding controllers in the 2DOF control scheme. Both controllers have a single adjustable parameter, respectively, which can be tuned in a monotonic manner to meet a good trade-off between control performance and robustness against process uncertainties. This merit can provide more flexibility for on-line tuning in comparison with previously developed control methods (e.g. the references ${ }^{24,26,36}$ ) based on numerical computation for controller parameterization. Moreover, the sufficient and necessary condition for holding robust stability of the closed-loop system against the plant model uncertainties is established and correspondingly, tuning constraints on these adjustable parameters are derived together with numerical guidelines for practical applications.

For clarity, the paper is organized as follows. Section 2 introduces the GP control structure together with process models adopted for control design. The proposed analytical controller design method is detailed in Section 3, addressing the load disturbance rejection and the set-point tracking, respectively. In Section 4, the sufficient and necessary condition for holding robust stability of the delayed closed-loop system is analyzed along with tuning constraints on the closed-loop controller for disturbance rejection. Four illustrative examples are shown in Section 5 to demonstrate the effectiveness and advantage of the proposed method. Finally, some conclusions are drawn in Section 6.

\section{Review of a generalized predictor-based control structure}

The GP based control structure given in the paper ${ }^{36}$ is shown in Figure 1, where $r$ and $y$ denote the set-point input and process output, respectively, and $w$ indicates an input load disturbance entering the process. The plant model is denoted by

$$
G(z)=G_{0}(z) z^{-d}=\frac{N(z)}{D(z)} z^{-d}
$$


where $G_{0}(z)$ indicates the delay-free part and $d$ is the time delay (assuming to be a multiple of the sampling period).

The filters $F_{1}(z)$ and $F_{2}(z)$ are used to yield the predicted undelayed output $\bar{y}$, while another filter, $F_{\mathrm{k}}(z)$, is included to eliminate steady output estimation error that may be provoked by external disturbance, model mismatch or process uncertainties. The controller denoted by $K_{\mathrm{f}}(z)$ is set to track the set-point, and $K(z)$ is the closed-loop controller for load disturbance rejection.

The undelayed output $\bar{y}(z)$ is predicted by using the current information of $u(z)$ and $y(z)$ in terms of the plant model, which can be derived as (see Figure 1)

$$
\bar{y}(z)=F_{1}(z) u(z)+F_{2}(z)[y(z)+n(z)]
$$

where $n(z)$ denotes measurement noise, $F_{1}(z)$ and $F_{2}(z)$ are filters configured in terms of the numerator and denominator of the plant model and its state-space formulation ${ }^{36}$ as

$$
\begin{gathered}
F_{1}(z)=\Phi(z, \lambda) \Gamma(z, \lambda) \\
F_{2}(z)=\frac{\tilde{N}^{*}(z, \lambda)}{(z-\lambda)^{m}}
\end{gathered}
$$

where

$$
\begin{gathered}
\Gamma(z, \lambda):=\frac{N(z)}{(z-\lambda)^{m}}, \quad|\lambda|<1 \\
G(z)=G_{0}(z) z^{-d}=\frac{N(z)}{D(z)} z^{-d}=\tilde{G}_{0}(z, \lambda) \Gamma(z, \lambda) z^{-d} \\
\tilde{G}_{0}(z, \lambda)=\frac{(z-\lambda)^{m}}{D(z)}=\frac{\tilde{N}(z, \lambda)}{D(z)}=C(\lambda)(z I-A)^{-1} B \\
\tilde{G}_{0}^{*}(z, \lambda):=C(\lambda)(z I-A)^{-1} A^{d} B=\frac{\tilde{N}^{*}(z, \lambda)}{D(z)} \\
\Phi(z, \lambda):=C(\lambda) \sum_{i=1}^{d} A^{i-1} B z^{-i}
\end{gathered}
$$

and $m$ is the number of zeros in $N(z),(A, B, C)$ is a minimum order state-space realization of $\widetilde{G}_{0}(z)$, and $C(\lambda)$ indicates an involvement with $\lambda$. 
Following the paper ${ }^{36}$, the prediction error filter, $F_{\mathrm{k}}(z)$, is configured as

$$
F_{\mathrm{k}}(z)=\frac{(1-\lambda)^{m}}{(z-\lambda)^{m}}
$$

Note that $\lambda$ is an adjustable parameter in $F_{1}(z), F_{2}(z), F_{\mathrm{k}}(z)$, It was shown that it can be tuned to balance the output prediction performance and its robustness against process uncertainties. Note that $m$ is the same as in (4).

An important advantage of the GP based control structure shown in Figure 1 is to remove the 'delay' out of the closed-loop such that a delay-free control design can be developed for the 'undelayed plant', as depicted in Figure 2. From this figure, the ideal transfer function relating $r$, $w$ and $n$ to $y$ can be derived as

$$
y(z)=\left[K_{\mathrm{f}}(z) \frac{K(z) G_{0}(z)}{1+K(z) G_{0}(z)} r(z)+\frac{G_{0}(z)}{1+K(z) G_{0}(z)} w(z)-\frac{K(z) G_{0}(z)}{1+K(z) G_{0}(z)} n(z)\right] z^{-d}
$$

The main controllers, $K_{\mathrm{f}}(z)$ and $K(z)$, however, were not specifically designed in the references $^{35,36}$, and no performance specifications or stability constraints were studied for engineering applications. To address these issues and obtain improved control performance, analytical designs for these two controllers will be proposed in the following sections.

In this paper, integrating and unstable processes with time delay are studied by means of the following two transfer function models often used in engineering practice.

$$
\begin{aligned}
G_{1}(z) & =\frac{k_{\mathrm{p}}\left(z-z_{0}\right)}{(z-1)\left(z-z_{\mathrm{p}}\right)} z^{-d} \\
G_{2}(z) & =\frac{k_{\mathrm{p}}\left(z-z_{0}\right)}{\left(z-z_{\mathrm{u}}\right)\left(z-z_{\mathrm{p}}\right)} z^{-d}
\end{aligned}
$$

where $\left|z_{\mathrm{p}}\right|<1$ and $\left|z_{\mathrm{u}}\right|>1$.

It is obvious that the discrete-time model in (12) describes an integrating process and the model in (13) represents an unstable process.

\section{Analytical controller design}

For clarity, the controller designs for the load disturbance rejection and the set-point tracking are detailed in the following two subsections, respectively. 


\subsection{Closed-loop controller for disturbance rejection}

In the GP control structure shown in Figure 1, $K(z)$ is the closed-loop controller for load disturbance rejection. The transfer functions between $\bar{y}, u$ and $w$ can be derived, respectively, as

$$
\begin{gathered}
\frac{\bar{y}(z)}{w(z)}=\left(1-\frac{K(z) G_{0}(z)}{1+K(z) G_{0}(z)}\right) G_{0}(z) \\
\frac{u(z)}{w(z)}=T_{d}(z)=\frac{K(z) G_{0}(z)}{1+K(z) G_{0}(z)}
\end{gathered}
$$

It is seen that the transfer function from $w$ to $u$ is exactly equivalent to the closed-loop complementary sensitivity function for the 'undelayed' system shown in Figure 2. In the ideal case, the load disturbance should be rejected immediately after being detected by the 'undelayed' closed-loop structure, i.e. $T_{\mathrm{d}}=z^{-1}$. However, practical constraints such as the closed-loop stability and actuator limits must be envisaged in engineering practice and should be taking into account in the controller design procedure.

To eliminate the output error arising from load disturbance while maintaining the internal stability of the closed-loop system, the following asymptotic tracking constraints must be satisfied,

$$
\begin{gathered}
\lim _{z \rightarrow 1}\left(1-T_{\mathrm{d}}\right)=0 \\
\lim _{z \rightarrow p_{i}}\left(1-T_{\mathrm{d}}\right)=0, \quad i=1,2, \ldots, l .
\end{gathered}
$$

where $p_{i}(i=1,2, \ldots, l$.$) are the process l$ transfer function poles on or outside the unit circle in the z-plane.

Note that for an integral process, the constraint in (17) should be substituted by

$$
\lim _{z \rightarrow 1} \frac{d}{d z}\left(1-T_{\mathrm{d}}\right)=0
$$

Based on a classification of the zero distribution in z-plane for the process models shown in (12) and (13), two cases are considered for the controller design, $\left|z_{0}\right|<1$ and $\left|z_{0}\right|>1$, as described below.

a) Minimum-phase (MP) plants 
When there is $\left|z_{0}\right|<1$ in the process model shown in (12) or (13),

$$
T_{\mathrm{d}}(z)=\left(\beta_{0}+\beta_{1} z+\cdots \beta_{l} z^{l}\right) \frac{\left(1-\lambda_{\mathrm{c}}\right)^{l+1}}{\left(z-\lambda_{\mathrm{c}}\right)^{l+1}}
$$

is proposed as the desired closed-loop transfer function from $w$ to $u$, where $\beta_{i}(i=1,2, \ldots, l$. $)$ are to be determined by the asymptotic constraints in (16)-(18), and $\lambda_{\mathrm{c}}$ is a tuning parameter for the closed-loop control performance.

Accordingly, the closed-loop controller can be inversely derived from (15) as

$$
K(z)=\frac{T_{\mathrm{d}}(z)}{1-T_{\mathrm{d}}(z)} \cdot \frac{1}{G_{0}(z)}
$$

Note that $K(z)$ may provoke inter-sample rippling in the output response or control signal if $K(z)$ has any pole with negative real part in $z$-plane, which arises from the corresponding zero of $G_{0}(z)$ as shown in (20). This is typically involved with the design of a discrete-time IMC controller'. To cope with the problem, it is proposed to build up the controller by

$$
\widehat{K}(z)=K(z) K_{\mathrm{q}}(z)
$$

where $K_{\mathrm{q}}(z)$ cancels all the poles of $K(z)$ with negative real part, i.e.

$$
K_{\mathrm{q}}(z)=z^{-n_{\mathrm{q}}} \prod_{j=1}^{n_{\mathrm{q}}} \frac{z-z_{j}}{1-z_{j}}
$$

where $z_{j}\left(j=1, \cdots n_{\mathrm{q}}\right)$ are the zeros with negative real part in $G_{0}(\mathrm{z})$, and $n_{\mathrm{q}}$ is the number of these zeros. Note that it follows $\hat{K}(z)=K(z)$ when $z \rightarrow 1$.

a1) Integrating processes

For an integrating process described by (12), there is $l=1$. It follows from (19) that

$$
T_{\mathrm{d}}(z)=\frac{\left(1-\lambda_{\mathrm{c}}\right)^{2}\left(\beta_{0}+\beta_{1} z\right)}{\left(z-\lambda_{\mathrm{c}}\right)^{2}}
$$

Substituting (23) into the constraints in (16) and (18) it yields

$$
\begin{aligned}
& \beta_{1}=\frac{2}{1-\lambda_{\mathrm{c}}} \\
& \beta_{0}=1-\beta_{1}
\end{aligned}
$$

If $z_{0}$ in (12) has no negative real part, substituting (12), (23)-(25) into (20) yields the 
closed-loop controller,

$$
K(z)=\frac{\left(1-\lambda_{\mathrm{c}}\right)\left(2 z-\lambda_{\mathrm{c}}-1\right)\left(z-z_{\mathrm{p}}\right)}{k_{\mathrm{p}}\left(z-z_{0}\right)(z-1)}
$$

In the case that $z_{0}$ has negative real part, it follows from (22) that

$$
K_{\mathrm{q}}=z^{-1} \frac{z-z_{0}}{1-z_{0}}
$$

Substituting (26) and (27) into (21) yields the corresponding controller that can avoid inter-sample rippling in the output response,

$$
\hat{K}(z)=\frac{\left(1-\lambda_{\mathrm{c}}\right)\left(2 z-\lambda_{\mathrm{c}}-1\right)\left(z-z_{\mathrm{p}}\right)}{k_{\mathrm{p}}\left(1-z_{0}\right) z(z-1)}
$$

a2) Unstable processes

For an unstable process described by (13), the desired closed-loop transfer function from $w$ to $u$ is the same as in (23), except for the coefficients in the numerator which should be derived by using the constraints in (16) and (17) as

$$
\begin{gathered}
\beta_{1}=\frac{\left(z_{\mathrm{u}}-\lambda_{\mathrm{c}}\right)^{2}-\left(1-\lambda_{\mathrm{c}}\right)^{2}}{\left(z_{\mathrm{u}}-1\right)\left(1-\lambda_{\mathrm{c}}\right)^{2}} \\
\beta_{0}=1-\beta_{1}
\end{gathered}
$$

If $z_{0}$ in (13) has no negative real part, substituting (13), (23), (29), (30) into (20) yields the closed-loop controller,

$$
K(z)=\frac{\left(1-\lambda_{\mathrm{c}}\right)^{2}\left(\beta_{1} z+\beta_{0}\right)\left(z-z_{\mathrm{u}}\right)\left(z-z_{\mathrm{p}}\right)}{k_{\mathrm{p}}\left(z-z_{0}\right)\left[\left(z-\lambda_{\mathrm{c}}\right)^{2}-\left(1-\lambda_{\mathrm{c}}\right)^{2}\left(\beta_{1} z+\beta_{0}\right)\right]}
$$

In the case that $z_{0}$ has negative real part, following a similar derivation as above, the closed-loop controller is obtained as

$$
\widehat{K}(z)=\frac{\left(1-\lambda_{\mathrm{c}}\right)^{2}\left(\beta_{1} z+\beta_{0}\right)\left(z-z_{\mathrm{u}}\right)\left(z-z_{\mathrm{p}}\right)}{k_{\mathrm{p}}\left(1-z_{0}\right) z\left[\left(z-\lambda_{\mathrm{c}}\right)^{2}-\left(1-\lambda_{\mathrm{c}}\right)^{2}\left(\beta_{1} z+\beta_{0}\right)\right]}
$$

Considering that the desired $K(z)$ (or $\widehat{K}(z)$ ) has an integrating function, i.e. having a pole at $z=1$, and substituting (29), (30) into (31)-(32), leads to $K(z)$ and $\widehat{K}(z)$ simplified, respectively, as 


$$
\begin{aligned}
& K(z)=\frac{\left(1-\lambda_{\mathrm{c}}\right)^{2}\left(\beta_{1} z+\beta_{0}\right)\left(z-z_{\mathrm{p}}\right)}{k_{\mathrm{p}}\left(z-z_{0}\right)(z-1)} \\
& \widehat{K}(z)=\frac{\left(1-\lambda_{\mathrm{c}}\right)^{2}\left(\beta_{1} z+\beta_{0}\right)\left(z-z_{\mathrm{p}}\right)}{k_{\mathrm{p}}\left(1-z_{0}\right) z(z-1)}
\end{aligned}
$$

b) Non-MP (NMP) plants

When there is $\left|z_{0}\right|>1$ in the process model shown in (12) or (13), the desired closed-loop transfer function from $w$ to $u$ is proposed as

$$
T_{\mathrm{d}}(z)=\left(\beta_{0}+\beta_{1} z+\cdots \beta_{l} z^{l}\right) \frac{\left(1-\lambda_{\mathrm{c}}\right)^{l+1}}{\left(z-\lambda_{\mathrm{c}}\right)^{l+1}} \frac{\left(1-z_{0}^{-1}\right)\left(z-z_{0}\right)}{\left(1-z_{0}\right)\left(z-z_{0}^{-1}\right)}
$$

where $\beta_{i}(i=1,2, \ldots, l$.$) are to be determined by the asymptotic constraints in (16)-(18), and \lambda_{\mathrm{c}}$ is a tuning parameter for the closed-loop control performance.

For an integrating process described by (12) with $\left|z_{0}\right|>1$, it follows from (35) that

$$
T_{\mathrm{d}}(z)=\frac{\left(1-\lambda_{\mathrm{c}}\right)^{2}\left(\beta_{0}+\beta_{1} z\right)\left(1-z_{0}^{-1}\right)\left(z-z_{0}\right)}{\left(z-\lambda_{\mathrm{c}}\right)^{2}\left(1-z_{0}\right)\left(z-z_{0}^{-1}\right)}
$$

Substituting (36) into the constraints in (16) and (18) it yields

$$
\begin{gathered}
\beta_{1}=\frac{2\left(1-z_{0}\right)-\left(1-\lambda_{\mathrm{c}}\right)\left(1+z_{0}\right)}{\left(1-\lambda_{\mathrm{c}}\right)\left(1-z_{0}\right)} \\
\beta_{0}=1-\beta_{1}
\end{gathered}
$$

Substituting (12), (36), (37) and (38) into (20) yields the closed-loop controller,

$$
K(z)=\frac{\left(1-z_{0}^{-1}\right)\left(1-\lambda_{\mathrm{c}}\right)^{2}\left(\beta_{1} z+\beta_{0}\right)\left(z-z_{\mathrm{p}}\right)}{k_{\mathrm{p}}\left(1-z_{0}\right)(z-1)\left[z-2 \lambda_{\mathrm{c}}-z_{0}^{-1}+2+\beta_{1}\left(1-\lambda_{\mathrm{c}}\right)^{2} / z_{0}\right]}
$$

For an unstable process described by (13) with $\left|z_{0}\right|>1$, the desired closed-loop transfer function from $w$ to $u$ is the same as in (36), except for the coefficients in the numerator which should be derived by using the constraints in (16) and (17) as

$$
\begin{gathered}
\beta_{1}=\frac{1}{z_{\mathrm{u}}-1}\left[\frac{\left(1-z_{0}\right)\left(z_{\mathrm{u}}-z_{0}^{-1}\right)\left(z_{\mathrm{u}}-\lambda_{\mathrm{c}}\right)^{2}}{\left(1-z_{0}^{-1}\right)\left(z_{\mathrm{u}}-z_{0}\right)\left(1-\lambda_{\mathrm{c}}\right)^{2}}-1\right] \\
\beta_{0}=1-\beta_{1}
\end{gathered}
$$

Substituting (13), (36), (40), (41) into (20) yields the closed-loop controller, 


$$
K(z)=\frac{\left(1-z_{0}^{-1}\right)\left(1-\lambda_{\mathrm{c}}\right)^{2}\left(\beta_{1} z+\beta_{0}\right)\left(z-z_{\mathrm{u}}\right)\left(z-z_{\mathrm{p}}\right)}{k_{\mathrm{p}}\left[\left(1-z_{0}\right)\left(z-z_{0}^{-1}\right)\left(z-\lambda_{\mathrm{c}}\right)^{2}-\left(1-\lambda_{\mathrm{c}}\right)^{2}\left(1-z_{0}^{-1}\right)\left(\beta_{1} z+\beta_{0}\right)\left(z-z_{0}\right)\right]}
$$

which can be simplified as

$$
K(z)=\frac{\left(1-z_{0}^{-1}\right)\left(1-\lambda_{\mathrm{c}}\right)^{2}\left(z-z_{\mathrm{p}}\right)}{k_{\mathrm{p}}(z-1)} Q(z)
$$

where

$$
Q(z)=\frac{\beta_{1} z+\beta_{0}}{\left(1-z_{0}\right)\left[z-2 \lambda_{\mathrm{c}}-z_{0}^{-1}+z_{\mathrm{u}}+1+\beta_{1}\left(1-\lambda_{\mathrm{c}}\right)^{2} / z_{0}\right]}
$$

\subsection{Set-point tracking controller}

$K(z)$ has been designed to obtain the desired closed-loop complementary sensitivity function $T_{\mathrm{d}}(z)$ in (15). It follows from (11) that the output / set-point transfer function is

$$
\frac{y(z)}{r(z)}=K_{\mathrm{f}}(z) \frac{K(z) G_{0}(z) z^{-d}}{1+K(z) G_{0}(z)}
$$

That is

$$
\frac{y(z)}{r(z)}=K_{\mathrm{f}}(z) T_{\mathrm{d}}(z) z^{-d}
$$

Assume $T_{\mathrm{d}}(z)$ being factorized into an all-pass part $T_{\mathrm{dA}}(z)$ and an MP part $T_{\mathrm{dM}}(z)$, i.e.

$$
T_{\mathrm{d}}(z)=T_{\mathrm{dA}}(z) T_{\mathrm{dM}}(z)
$$

According to the IMC theory ${ }^{9}$ for set-point tracking, an ideal controller should take the following form to satisfy the $\mathrm{H}_{2}$ optimal control performance,

$$
K_{\mathrm{fl}}(z)=\left(z^{n_{\mathrm{g}}} T_{\mathrm{dM}}(z)\right)^{-1}
$$

where $n_{\mathrm{g}}$ is a positive integer chosen to keep $z^{n_{\mathrm{g}}} T_{\mathrm{dM}}(z)$ bi-proper, i.e. its numerator and denominator have the same degree.

For practical application, it is proposed to add a low-pass filter to the above ideal controller so that the control peak can be modulated to comply with the controller capacity in practice, i.e.

$$
K_{\mathrm{f} 2}(z)=\frac{\left(1-\lambda_{\mathrm{f}}\right)^{n_{\mathrm{f}}} z^{n_{\mathrm{f}}}}{\left(z-\lambda_{\mathrm{f}}\right)^{n_{\mathrm{f}}}}
$$

where $n_{\mathrm{f}}$ is the filter order that may be specified by the user, and $\lambda_{\mathrm{f}}$ is a tuning parameter. 
When $\lambda_{\mathrm{f}} \rightarrow 0$, then $K_{\mathrm{f} 2}(z)=1$. Obviously, the simplest form is a first-order filter,

$$
K_{\mathrm{f} 2}(z)=\frac{\left(1-\lambda_{\mathrm{f}}\right) z}{z-\lambda_{\mathrm{f}}}
$$

Therefore, the set-point tracking controller is obtained as

$$
K_{\mathrm{f}}(z)=K_{\mathrm{f} 1}(z) K_{\mathrm{f} 2}(z)
$$

where the tuning parameter, $\lambda_{\mathrm{f}}$, can be tuned to reach a compromise between the set-point tracking performance and the control effort. With a smaller value of $\lambda_{\mathrm{f}}$, the set-point tracking speed will be faster, but the required output energy of $K_{\mathrm{f}}$ is larger. In addition, the set-point response will become more aggressive in the presence of process uncertainties; on the contrary, if $\lambda_{\mathrm{f}}$ is tuned to be larger, the nominal set-point tracking speed will becomes slower but the output energy of $K_{\mathrm{f}}$ is alleviated, and correspondingly, the set-point response will become less sensitive to process uncertainties. Note that when $z \rightarrow 1, K_{\mathrm{f}}$ recovers the optimality, i.e. $K_{\mathrm{f}}(z)=K_{\mathrm{f} 1}(z)$.

Substituting (48), (50) and (51) into (46) the obtained output response is

$$
y(z)=z^{-n_{\mathrm{g}}-d} K_{\mathrm{f} 2}(z) T_{\mathrm{dA}}(z) r(z)
$$

For instance, in the case of $T_{\mathrm{dA}}(z)=1$ and $n_{\mathrm{g}}=1$, using a first-order filter in (50) yields the output response in the form of

$$
y(z)=\frac{1-\lambda_{\mathrm{f}}}{z-\lambda_{\mathrm{f}}} z^{-d} r(z)
$$

By using the inverse z-transform, the set-point response to a step change in time domain can be derived as

$$
y\left(k T_{\mathrm{s}}\right)= \begin{cases}0, & k \leq d \\ 1-\lambda_{\mathrm{f}}^{(k-d)}, & k>d\end{cases}
$$

where $T_{\mathrm{s}}$ denotes the sampling period. It is seen that there is no overshoot in the set-point response and its time domain specifications can be quantitatively tuned by $\lambda_{\mathrm{f}}$. For example, consider the rise time, denoted by $t_{\mathrm{r}}$, which is usually defined as the time when the output response first reaches $90 \%$ of its final steady-state value in response to a step change of the set-point. If $t_{\mathrm{r}}=15(\mathrm{~s})$ is specified for a process with $d=20$ and $T_{\mathrm{s}}=0.2(\mathrm{~s})$, it can be 
computed from (54) that the filter parameter should be taken as $\lambda_{\mathrm{f}}=0.959$.

To sum up, all the controller formulae for different cases of the process models are listed in Table 1. For the convenience of practical application, the proposed control design procedure is summarized as follows.

(i) Build up a GP structure by configuring the stable filters $F_{1}(z), F_{2}(z)$, and $F_{\mathrm{k}}(z)$, according to (2), (3), (10), with a choice of $|\lambda|<1$;

(ii) Design the disturbance rejection controller $K(z)$ for different cases of the process models as listed in Table 1;

(iii) Monotonically tune the single adjustable parameter $\lambda_{\mathrm{c}}$ in $K(z)$, in combination with a retuning of $\lambda$ in $F_{1}(z), F_{2}(z)$ and $F_{k}(z)$ for improving output prediction if necessary, to achieve a good trade-off between disturbance rejection performance and closed-loop robust stability.

(iv) Factorize the resultant closed-loop sensitivity function $T_{\mathrm{d}}(z)$ as shown in (23) or (36), and then design the set-point tracking controller $K_{\mathrm{f}}(z)$ shown in (51). Then tune the single adjustable parameter $\lambda_{\mathrm{f}}$ in $K_{\mathrm{f}}(z)$ to achieve the desired set-point tacking performance and its robustness against process uncertainties.

\section{Robust stability analysis}

Considering the uncertainties of the original delayed process being described in a multiplicative form, $\Delta(z)=[P(z)-G(z)] / G(z)$, as shown in Figure 3, we reformulate the closed-loop system is reformulated in the standard $M-\Delta$ form shown in Figure 4 for robust stability analysis. The transfer function from the output $v_{\text {out }}$ to the input $v_{\text {in }}$ of $\Delta(z)$ can be derived as

$$
M=\frac{v_{\text {in }}}{v_{\text {out }}}=H_{2} \frac{K G_{0} z^{-d}}{1+K G_{0}}
$$

where

$$
H_{2}=F_{2} F_{k} z^{-d}-F_{2}-F_{k}=\frac{(1-\lambda)^{m} \tilde{N}^{*}(z) z^{-d}-(z-\lambda)^{m}\left[\tilde{N}^{*}(z)+(1-\lambda)^{m}\right]}{(z-\lambda)^{2 m}}
$$

According to the small gain theorem (see e.g. the reference ${ }^{9}$ ), the closed-loop structure holds 
robust stability in the presence of process uncertainty if and only if

$$
\left\|H_{2} \frac{K G_{0} z^{-d}}{1+K G_{0}} \Delta\right\|_{\infty}<1
$$

For integrating and unstable processes described by (12) and (13), we take $m=1$ in (10) for output prediction. It can be derived using (3) and (4) that

$$
H_{2}=\frac{(1-\lambda)\left(\alpha_{1} z+\alpha_{0}\right) z^{-d}-(z-\lambda)\left(\alpha_{1} z+\alpha_{0}\right)-(1-\lambda)(z-\lambda)}{(z-\lambda)^{2}}
$$

where $\alpha_{0}$ and $\alpha_{1}$ are determined from

$$
F_{2}(z-\lambda)=\sum_{i=0}^{m} \alpha_{m} z^{m}
$$

When there is $\left|z_{0}\right|<1$ in the process model shown in (12) or (13), by substituting (15), (23) and (58) into (57), the robust stability condition is obtained as

$$
\left\|\frac{\left(1-\lambda_{\mathrm{c}}\right)^{2}\left[\rho_{0} z^{3}+\rho_{1} z^{2}+\rho_{2} z+\rho_{3}+\rho_{4} z^{-d}+\rho_{5} z^{-(d-1)}+\rho_{6} z^{-(d-2)}\right]}{\left(z-\lambda_{\mathrm{c}}\right)^{2}(z-\lambda)^{2}}\right\|_{\infty}<\frac{1}{\|\Delta(z)\|_{\infty}}
$$

where $\rho_{0}=-\alpha_{1} \beta_{1}, \quad \rho_{1}=\beta_{1}\left(\lambda \alpha_{1}-\alpha_{0}-1+\lambda\right)-\beta_{0} \alpha_{1}, \quad \rho_{2}=\beta_{1}\left(1-\lambda+\alpha_{0}\right) \lambda+\beta_{0}\left(\lambda \alpha_{1}-\alpha_{0}-1+\lambda\right)$, $\rho_{3}=\beta_{0} \lambda\left(\alpha_{0}+1-\lambda\right), \rho_{4}=\beta_{0} \alpha_{0}(1-\lambda), \quad \rho_{5}=(1-\lambda)\left(\beta_{1} \alpha_{0}+\beta_{0} \alpha_{1}\right), \quad \rho_{6}=(1-\lambda) \beta_{1} \alpha_{1}$.

For the case of $\left|z_{0}\right|>1$ in the process model shown in (12) or (13), it can be verified by substituting (15), (36) and (58) into (57) that the robust stability condition is the same as (60).

Consider the following three types of model uncertainty that are often adopted for assessment in engineering practice,

$$
\begin{aligned}
& \Delta=\frac{\Delta k_{\mathrm{p}}}{k_{\mathrm{p}}} \\
& \Delta=z^{-\Delta d}-1 \\
& \Delta=\left(1+\frac{\Delta k_{\mathrm{p}}}{k_{\mathrm{p}}}\right) z^{-\Delta d}-1
\end{aligned}
$$

Since a rational $z$-transform (i.e. $z=e^{j \omega T_{s}}$ ) is a periodic function with respect to $\omega$, by defining $z=e^{j \theta} \quad(0<\theta<2 \pi)$ and substituting (61)-(63) into (60), respectively, the robust stability constraints are correspondingly derived as 


$$
\begin{gathered}
\frac{\left(1-\lambda_{\mathrm{c}}\right)^{2} \sqrt{x_{1}^{2}+x_{2}^{2}}}{\sqrt{x_{3}^{2}+x_{4}^{2}}}<\frac{k_{\mathrm{p}}}{\Delta k_{\mathrm{p}}} \\
\frac{\left(1-\lambda_{\mathrm{c}}\right)^{2} \sqrt{x_{1}^{2}+x_{2}^{2}}}{\sqrt{x_{3}^{2}+x_{4}^{2}}}<\frac{1}{\sqrt{(\cos \Delta d \theta-1)^{2}+(\sin \Delta d \theta)^{2}}} \\
\frac{\left(1-\lambda_{\mathrm{c}}\right)^{2} \sqrt{x_{1}^{2}+x_{2}^{2}}}{\sqrt{x_{3}^{2}+x_{4}^{2}}}<\frac{1}{\sqrt{\left[\left(1+\frac{\Delta k}{k}\right) \cos \Delta d \theta-1\right]^{2}+\left[\left(1+\frac{\Delta k}{k}\right) \sin \Delta d \theta\right]^{2}}}
\end{gathered}
$$

where

$$
\begin{gathered}
x_{1}=\rho_{6} \cos (d-2) \theta+\rho_{5} \cos (d-1) \theta+\rho_{4} \cos d \theta+\rho_{0} \cos 3 \theta+\rho_{1} \cos 2 \theta+\rho_{2} \cos \theta+\rho_{3} \\
x_{2}=-\rho_{6} \sin (d-2) \theta-\rho_{5} \sin (d-1) \theta-\rho_{4} \sin d \theta+\rho_{0} \sin 3 \theta+\rho_{1} \sin 2 \theta+\rho_{2} \sin \theta \\
x_{3}=\cos 4 \theta-2\left(\lambda_{\mathrm{c}}+\lambda\right) \cos 3 \theta+\left(\lambda_{\mathrm{c}}{ }^{2}+\lambda^{2}+4 \lambda_{\mathrm{c}} \lambda\right) \cos 2 \theta-2\left(\lambda_{\mathrm{c}}{ }^{2} \lambda+\lambda \lambda_{\mathrm{c}}{ }^{2}\right) \cos \theta+\lambda^{2} \lambda_{\mathrm{c}}{ }^{2} \\
x_{4}=\sin 4 \theta-2\left(\lambda_{\mathrm{c}}+\lambda\right) \sin 3 \theta+\left(\lambda_{\mathrm{c}}{ }^{2}+\lambda^{2}+4 \lambda_{\mathrm{c}} \lambda\right) \sin 2 \theta-2\left(\lambda_{\mathrm{c}}{ }^{2} \lambda+\lambda \lambda_{\mathrm{c}}{ }^{2}\right) \sin \theta
\end{gathered}
$$

It is seen that all the above robust stability constraints are nonlinear inequalities with respect to the filter parameter, $\lambda$, and the closed-loop controller parameter, $\lambda_{\mathrm{c}}$. Generally, both $\lambda$ and $\lambda_{\mathrm{c}}$ may be initially taken in the interval of $[0.8,0.99]$ to maintain the closed-loop stability. To improve the control performance or closed-loop stability, it is suggested to monotonically decrease $\lambda$ on line in order to improve the output prediction performance but in exchange for its robustness against process uncertainties, and vice versa. Similarly, $\lambda_{\mathrm{c}}$ can be monotonically tuned on line to meet a good trade-off between the disturbance rejection performance and the closed-loop robust stability. As the above robust stability constraints cannot be solved analytically for tuning $\lambda_{\mathrm{c}}$, numerical tuning guidelines can be explored for practical applications, which will be illustrated by simulation examples shown in Section 5. Based on the simulation study, it is suggested to tune $\lambda_{\mathrm{c}}$ in the interval of [0.9 0.99] to obtain a good trade-off between the closed-loop control performance and its robust stability for integrating and unstable processes with time delay.

Remark 1. The robust stability constraint shown in (57) can be used for different designs on the closed-loop controller as discussed in the paper ${ }^{36}$. With the proposed controller design, it is seen 
from the corresponding stability constraints shown in (64)-(66) that these constraints are in fact inequalities with respect to the tuning parameter, $\lambda_{\mathrm{c}}$, in the closed-loop controller, $K$. Therefore, it is convenient to check these constraints in tuning $\lambda_{\mathrm{c}}$ for a good trade-off between the closed-loop control performance and its robust stability. Note that these constraints may also be used to evaluate an allowable upper bound of the process uncertainties.

\section{Simulation study}

Four benchmark examples studied in the references are used for illustration, respectively for two MP integrating processes, an NMP integrating process, and an unstable process. To assess control performance, the commonly used performance index of integral-absolute-error (IAE) of process output is adopted which should be as small as possible.

Example 1. Consider the integrating process with time delay studied in the reference ${ }^{36}$,

$$
G(s)=\frac{e^{-4 s}}{s(s+1)}
$$

With a sampling period of $T_{\mathrm{s}}=0.2(\mathrm{~s})$, a discrete-time model of the process was there ${ }^{36}$ obtained

$$
G(z)=\frac{0.018731(z+0.9335)}{(z-1)(z-0.8187)} z^{-20}
$$

The filters $F_{1}(z), F_{2}(z), F_{\mathrm{k}}(z)$ are configured based on the design formulae in (3), (4), (10) with a choice of $\lambda=0.84$ as done in the reference ${ }^{36}$, i.e.

$$
F_{1}(z)=\Phi \Gamma
$$

where $\Phi$ is in the form of (9) with $d=20, A=\left[\begin{array}{cc}1.8187 & -0.8187 \\ 1 & 0\end{array}\right], B=\left[\begin{array}{l}1 \\ 0\end{array}\right]$ and $C=\left[\begin{array}{ll}1 & -0.84\end{array}\right]$,

$$
\begin{gathered}
\Gamma=\frac{0.01873 z+0.01752}{z-0.84} \\
F_{2}(z)=\frac{0.8848 z-0.7248}{z-0.84} \\
F_{\mathrm{k}}(z)=\frac{0.16}{z-0.84}
\end{gathered}
$$


Now let us consider the analytical controllers proposed in section 3. Note that there is a zero $\left(z_{0}=-0.9335\right)$ of $G(z)$ with negative real part. The disturbance rejection controller is therefore obtained by employing the controller design formula in (28) as

$$
K(z)=\frac{\left(1-\lambda_{\mathrm{c}}\right)(z-0.8187)\left(2 z-\lambda_{\mathrm{c}}-1\right)}{0.03622 z(z-1)}
$$

The proposed controller design formulae in (23)-(25) and (47)-(51) give the set-point tracking controller,

$$
K_{\mathrm{f}}(z)=\frac{\left(z-\lambda_{\mathrm{c}}\right)^{2}\left(1-\lambda_{\mathrm{f}}\right)}{\left(1-\lambda_{\mathrm{c}}\right)^{2}\left(\beta_{0}+\beta_{1} z\right)\left(z-\lambda_{\mathrm{f}}\right)}
$$

where $\beta_{1}=2 /\left(1-\lambda_{\mathrm{c}}\right)$ and $\beta_{0}=1-\beta_{1}$.

For illustration, a unity step change is added to the system input at $t=0(\mathrm{~s})$ and a step load disturbance with a magnitude of -0.1 is added to the process input at $t=80(\mathrm{~s})$. By taking $\lambda_{\mathrm{c}}=0.973$ and $\lambda_{\mathrm{f}}=0.942$ in the above controllers to obtain a similar rising speed of the set-point response and a similar disturbance response peak with those of the reference ${ }^{36}$ for comparison, the control results are shown in Figure 5 along with the IAE indices listed in Tables 2 and 3. It is seen that both the set-point response and disturbance response have been apparently improved by the proposed method.

Now assume that the process gain is actually $20 \%$ larger and process time delay is $20 \%$ smaller than the model. The perturbed system responses are shown in Figure 6 along with the IAE indices listed in Tables 2 and 3 . It is seen that the proposed method well maintains the performance in the presence of these process uncertainties.

The control performance variation for the process by tuning $\lambda_{\mathrm{c}}$ is illustrated in Figure 7 , where $T_{\mathrm{d}}$ is the closed-loop complementary sensitivity function shown in (23) and 'Peak' denotes the disturbance response peak to a step load disturbance with a magnitude of 0.5 . It is seen that when $\lambda_{\mathrm{c}}$ is tuned to a larger value, a smaller value of $\left|T_{\mathrm{d}}\right|$ is obtained which indicates better closed-loop robust stability but in exchange for the disturbance rejection performance. On the contrary, tuning $\lambda_{\mathrm{c}}$ to a smaller value will result in enhanced disturbance rejection performance but degrade the closed-loop robust stability. 
Numerical relationship between the output response peak to a step change of load disturbance $(w)$ and the model parameter $z_{\mathrm{p}} \in(-1,1)$ as shown in (12) is plotted in Figure 8 with respect to tuning $\lambda_{\mathrm{c}}$ in the intervals of $[0.05,0.95]$ and $[0.95,0.999]$, respectively. It is seen from Figure 8(a) that the disturbance response peak is increased with respect to $z_{\mathrm{p}} \in[0,0.99]$, but remains almost the same for $z_{\mathrm{p}} \in[-0.99,0]$, regardless of tuning $\lambda_{\mathrm{c}}$ in the interval of $[0.05,0.95]$. When $\lambda_{\mathrm{c}}$ is tuned to be larger than 0.99 , the disturbance response peak will become evidently larger as shown in Figure 8(b). Moreover, Figure 9 shows the numerical relationship between the disturbance response peak and $\lambda_{\mathrm{c}}$ regarding different values of the process time delay. It is seen that tuning $\lambda_{\mathrm{c}}$ is limited in the range of $(0,1)$ for such an integral process, and different values of $\lambda_{c} \in(0,0.9)$ gives similar control results, except for $\lambda_{\mathrm{c}} \in(0.9,1)$. Obviously, when $\lambda_{\mathrm{c}} \rightarrow 1$, the peak will become larger, and moreover, when the time delay is larger, the peak will become larger.

Example 2. Consider the integrating process with time delay studied in the reference ${ }^{25}$,

$$
G(s)=\frac{e^{-6.567 s}}{s(3.4945 s+1)}
$$

With a sampling period of $T_{\mathrm{s}}=0.2(\mathrm{~s})$, a discrete-time model of the process is obtained as

$$
G(z)=\frac{0.005616(z+0.9811)}{(z-1)(z-0.9444)} z^{-33}
$$

The filters $F_{1}(z), F_{2}(z), F_{\mathrm{k}}(z)$, designed as proposed in reference ${ }^{36}$ are configured based on the design formulae in (3), (4), (10) with a choice of $\lambda=0.9672$, i.e.

$$
F_{1}(z)=\Phi \Gamma
$$

where $\Phi$ is in the form of (9) with $d=33, A=\left[\begin{array}{cc}1.9444 & -0.9444 \\ 1 & 0\end{array}\right], B=\left[\begin{array}{l}1 \\ 0\end{array}\right]$, and $C=\left[\begin{array}{ll}1 & -0.9672\end{array}\right]$

$$
\begin{gathered}
\Gamma=\frac{0.005616 z+0.00551}{z-0.9672} \\
F_{2}(z)=\frac{0.6515 z-0.6187}{z-0.9672} \\
F_{\mathrm{k}}(z)=\frac{0.03278}{z-0.9672}
\end{gathered}
$$


Note that there is a zero $\left(z_{0}=-0.9811\right)$ of $G(z)$ with negative real part. The disturbance rejection controller is therefore obtained by employing the controller design formula in (28) as

$$
K(z)=\frac{\left(1-\lambda_{\mathrm{c}}\right)(z-0.9444)\left(2 z-\lambda_{\mathrm{c}}-1\right)}{0.011126 z(z-1)}
$$

The proposed controller design formulae in (23)-(25) and (47)-(51) give the set-point tracking controller,

$$
K_{\mathrm{f}}(z)=\frac{\left(z-\lambda_{\mathrm{c}}\right)^{2}\left(1-\lambda_{\mathrm{f}}\right)^{2}}{\left(1-\lambda_{\mathrm{c}}\right)^{2}\left(\beta_{0}+\beta_{1} z\right)\left(z-\lambda_{\mathrm{f}}\right)^{2}}
$$

where $\beta_{1}=2 /\left(1-\lambda_{\mathrm{c}}\right)$ and $\beta_{0}=1-\beta_{1}$.

For illustration, a unity step change is added to the system input at $t=0(\mathrm{~s})$ and a step load disturbance with a magnitude of -0.1 is added to the process input at $t=100(\mathrm{~s})$. To make comparison with that of the reference ${ }^{25}$, we take $\lambda_{\mathrm{c}}=0.94$ and $\lambda_{\mathrm{f}}=0.96$ in the above controllers for illustration. The control results are shown in Figure 10 along with the IAE indices listed in Tables 2 and 3. It is seen that both the set-point response and disturbance response have been improved by the proposed method.

Now assume that the process gain is actually $20 \%$ smaller and process time delay is $20 \%$ larger than the model. Figure 11 shows the perturbed system responses, and the corresponding IAE indices are listed in Tables 2 and 3. It is seen that the proposed method maintains good robust stability, while the reference ${ }^{25}$ could not hold the closed-loop stability any longer.

Example 3. Consider the NMP integrating process with time delay studied in the references $^{37,38}$,

$$
G(s)=0.547 \frac{(-0.418 s+1) e^{-0.1 s}}{s(1.06 s+1)}
$$

With a sampling period of $T_{\mathrm{s}}=0.1(\mathrm{~s})$, a discrete-time model of the process is obtained as

$$
G(z)=\frac{0.01808(z-1.2727)}{(z-1)(z-0.91)} z^{-1}
$$

Again, the filters $F_{1}(z), F_{2}(z), F_{\mathrm{k}}(z)$ are configured based on the design formulae in (3), (4), (10) with a choice of $\lambda=0.98$, i.e.

$$
F_{1}(z)=\Phi \Gamma
$$


where $\Phi$ is in the form of (9) with $d=1, A=\left[\begin{array}{cc}1.9613 & -0.9608 \\ 1 & 0\end{array}\right], B=\left[\begin{array}{l}1 \\ 0\end{array}\right]$ and $C=\left[\begin{array}{ll}1 & -0.98\end{array}\right]$

$$
\begin{gathered}
\Gamma=\frac{-0.01808 z+0.023}{z-0.98} \\
F_{2}(z)=\frac{0.8663 z-0.8463}{z-0.98} \\
F_{\mathrm{k}}(z)=\frac{0.02}{z-0.98}
\end{gathered}
$$

The disturbance rejection controller is obtained by employing the controller design formulae in (39) as

$$
K(z)=\frac{0.2143\left(1-\lambda_{\mathrm{c}}\right)^{2}\left(\beta_{1} z+\beta_{0}\right)(z-0.91)}{0.00493(z-1)\left(z+1.2143-2 \lambda_{\mathrm{c}}+0.7857 \beta_{1}\left(1-\lambda_{\mathrm{c}}\right)^{2}\right)}
$$

The proposed controller design formulae in (36)-(38) and (47)-(51) give the set-point tracking controller,

$$
K_{\mathrm{f}}(z)=\frac{\left(z-\lambda_{\mathrm{c}}\right)^{2}\left(1-\lambda_{\mathrm{f}}\right)}{\left(1-\lambda_{\mathrm{c}}\right)^{2}\left(\beta_{0}+\beta_{1} z\right)\left(z-\lambda_{\mathrm{f}}\right)}
$$

where

$$
\begin{gathered}
\beta_{1}=\frac{2\left(1-z_{0}\right)-\left(1-\lambda_{\mathrm{c}}\right)\left(1+z_{0}\right)}{\left(1-\lambda_{\mathrm{c}}\right)\left(1-z_{0}\right)} \\
\beta_{0}=1-\beta_{1} .
\end{gathered}
$$

Note that an IMC based PID control method was given in the reference ${ }^{37}$, which had shown improved control performance compared with that of the reference ${ }^{38}$. For fair comparison, the 2DOF IMC control method presented in Chapter 8 of the bibliography ${ }^{2}$ is used here, of which the control structure had been studied in the references ${ }^{10,11}$. The corresponding controllers for set-point tracking and load disturbance rejection are obtained, respectively, as

$$
\begin{gathered}
C_{\mathrm{s}}=\frac{\mathrm{s}(1.06 \mathrm{~s}+1)}{0.547(0.418 s+1)\left(\gamma_{\mathrm{s}} \mathrm{s}+1\right)} \\
C_{\mathrm{f}}=\frac{T_{\mathrm{d}}}{G\left(1-T_{\mathrm{d}}\right)}
\end{gathered}
$$




$$
T_{\mathrm{d}}=\frac{\left(a_{1} s+1\right)(-0.418 s+1)}{\left(\gamma_{\mathrm{f}} s+1\right)^{2}(0.418 s+1)} e^{-0.1 s}
$$

together with the desired transfer function for set-point response,

$$
\begin{gathered}
T_{\mathrm{r}}=C_{\mathrm{s}} G=\frac{(-0.418 s+1)}{(0.418 s+1)\left(\gamma_{\mathrm{s}} s+1\right)} e^{-0.1 s} \\
a_{1}=2 \gamma_{\mathrm{f}}+\theta+0.836
\end{gathered}
$$

where $\gamma_{\mathrm{s}}$ and $\gamma_{\mathrm{f}}$ are the tuning parameters of $C_{\mathrm{s}}$ and $C_{\mathrm{f}}$, respectively.

For illustration, a unity step change is added to the system input at $t=0(\mathrm{~s})$ and a step load disturbance with a magnitude of -0.5 is added to the process input at $t=40$ (s). Taking $\lambda_{\mathrm{c}}=0.93$ and $\lambda_{\mathrm{f}}=0.92$ in the proposed controllers and $\gamma_{\mathrm{s}}=1.5, \gamma_{\mathrm{f}}=1.9$ in the above controllers of the bibliography ${ }^{2}$ to obtain a similar rising speed of the set-point response and a similar recovery time of the load disturbance response with those of the reference ${ }^{37}$ for comparison, the control results are shown in Figure 12 along with the IAE indices listed in Tables 2 and 3. It is seen that both the set-point response and disturbance response have been evidently improved by the proposed method.

Now assume that the process gain is actually $20 \%$ larger and the process time delay is twice larger. The perturbed system responses are shown in Figure 13, and the corresponding IAE indices are listed in Tables 2 and 3. It is again seen that the proposed method well maintains the response stability against process uncertainties.

Example 4. Consider the unstable process with time delay studied in the references ${ }^{26,36}$,

$$
G(s)=\frac{2 e^{-5 s}}{(10 s-1)(2 s+1)}
$$

With a sampling period of $T_{\mathrm{s}}=0.1(\mathrm{~s})$, a discrete-time model of the process was obtained in the reference ${ }^{36}$ as

$$
G(z)=\frac{0.00049342(z+0.9868)}{(z-1.0046)(z-0.9564)} z^{-50}
$$

The filters $F_{1}(z), F_{2}(z), F_{\mathrm{k}}(z)$ are configured based on the design formulae in (3), (4), (10) with a choice of $\lambda=0.98$ as proposed in the reference ${ }^{36}$, i.e. 


$$
F_{1}(z)=\Phi \Gamma
$$

where $\Phi$ is in the form of (9) with $d=50, A=\left[\begin{array}{cc}1.9613 & -0.9608 \\ 1 & 0\end{array}\right], B=\left[\begin{array}{l}1 \\ 0\end{array}\right]$ and $C=\left[\begin{array}{ll}1 & -0.98\end{array}\right]$

$$
\begin{gathered}
\Gamma=\frac{0.00049342 z+0.00048691}{z-0.98} \\
F_{2}(z)=\frac{0.8824 z-0.8418}{z-0.98} \\
F_{\mathrm{k}}(z)=\frac{0.02}{z-0.98}
\end{gathered}
$$

The disturbance rejection controller is obtained by using the controller design formulae in (34) as

$$
K(z)=\frac{\left(1-\lambda_{\mathrm{c}}\right)^{2}\left(\beta_{1} z+\beta_{0}\right)(z-0.9564)}{0.00098033 z(z-1)}
$$

The proposed controller design formulae in (23), (29), (30) and (47)-(51) give the set-point tracking controller,

$$
K_{\mathrm{f}}=K_{\mathrm{f} 1}(z) K_{\mathrm{f} 2}(z)=\frac{\left(1-\lambda_{\mathrm{f}}\right)^{2} z\left(z-\lambda_{\mathrm{c}}\right)^{2}}{\left(1-\lambda_{\mathrm{c}}\right)^{2}\left(z-\lambda_{\mathrm{f}}\right)^{2}\left(\beta_{1} z+\beta_{0}\right)}
$$

where $\beta_{1}=\left[\left(1.0046-\lambda_{\mathrm{c}}\right)^{2}-\left(1-\lambda_{\mathrm{c}}\right)^{2}\right] /\left[0.0046\left(1-\lambda_{\mathrm{c}}\right)^{2}\right]$ and $\beta_{0}=1-\beta_{1}$.

For illustration, a unity step change is added to the system input at $t=0(\mathrm{~s})$ and a step load disturbance with a magnitude of -0.1 is added to the process input at $t=150$ (s). The control results compared with the references ${ }^{26,36}$ are shown in Figure 14 along with the corresponding IAE indices listed in Tables 2 and 3. It is seen that given the similar set-point response, improved disturbance rejection performance is obtained by the proposed method.

Then assume that all the process parameters are actually $20 \%$ larger than the model, the perturbed system responses are shown in Figure 15, and the corresponding IAE indices are listed in Tables 2 and 3. It is once again seen that the proposed method maintains good robust stability.

Based on the process model, numerical relationship between the disturbance response peak and $\lambda_{\mathrm{c}}$ is plotted in Figure 16 regarding different values of the process time delay. It is seen that for such an unstable process, the trend of the disturbance response peak is similar to that of an 
integrating process like example 1 . The tuning range of $\lambda_{\mathrm{c}}$, however, becomes significantly narrower with the increment of time delay or $z_{\mathrm{p}}$ in the process model as shown in (13).

\section{Conclusions}

An analytical design of a 2DOF control structure using a GP has been proposed for integrating and unstable systems with long time delay, to overcome the deficiency of numerical computation for controller parameterization that is case-dependent and inconvenient for on-line tuning as involved when using recently developed predictor-based control methods. Both the set-point tracking response and the load disturbance rejection response can be tuned separately in a monotonic manner through a single adjustable parameter. Based on a classification of the plant model zero/pole distribution in the $z$-plane, controller design formulae have been explicitly derived for different cases, while addressing the problem of inter-sample rippling provoked by the model zero having negative real part. Analytical expression for the set-point tracking response has been derived based on the plant model and the controller formula, therefore facilitating the assessment of quantitative performance specifications of the set-point response in engineering practice. The closed-loop controller for disturbance rejection has been analytically derived by proposing a desired transfer function between the process input and the load disturbance. The sufficient and necessary condition for holding the closed-loop robust stability has been analyzed, and correspondingly, robust tuning constraints on the closed-loop controller have been derived in terms of different model uncertainty forms, along with numerical tuning guidelines explored based on illustrative examples with a wide range of model parameters. The applications to benchmark examples from the recent references ${ }^{24,26,35,36}$, where numerical controller design methods rather than analytical formulae were used, have well demonstrated the effectiveness and merit of the proposed method.

\section{Acknowledgement}

This work is supported in part by the National Thousand Talents Program of China, NSF China Grants 61473054, and the Fundamental Research Funds for the Central Universities of China. 


\section{Reference}

[1] Normey-Rico, J. E. Control of dead-time processes. Springer, London UK, 2007.

[2] Liu, T.; Gao, F. Industrial Process Identification and Control Design: Step-test and Relay-Experiment-Based Methods. Springer, London UK, 2012.

[3] Mataušek, M. R.; Šekara, T. B. PID controller frequency-domain tuning for stable, integrating and unstable processes, including dead-time. Journal of Process Control 2011, 21(1), 17-27.

[4] Ribić, A. I.; Mataušek, M. R. A dead-time compensating PID controller structure and robust tuning. Journal of Process Control 2012, 22(7), 1340-1349.

[5] Normey-Rico, J. E.; Julio, E.; Guzman, J. L. Unified PID tuning approach for stable, integrative and unstable dead-time processes. Industrial \& Engineering Chemistry Research 2013, 52(47), 16811-16819.

[6] Vanavil, B.; Anusha, A.; Perumalsamy, M. et al, Enhanced IMC-PID controller design with lead-lag filter for unstable and integrating processes with time delay. Chemical Engineering Communications 2014, 201(11), 1468-1496.

[7] Panda, R. C. Synthesis of PID controller for unstable and integrating processes. Chemical Engineering Science 2009, 64(12), 2807-2816.

[8] Vanavil, B.; Chaitanya, K. K.; Rao, A. S. Improved PID controller design for unstable time delay processes based on direct synthesis method and maximum sensitivity. International Journal of Systems Science 2015, 46(8), 1349-1366.

[9] Morari, M.; Zafiriou, E. Robust process control. New Jersey, Prentice Hall, 1989.

[10] Liu, T.; Zhang, W.; Gu, D. Analytical design of two-degree-of-freedom control scheme for open-loop unstable processes with time delay. Journal of Process Control 2005, 15(5), $559-572$.

[11] Liu, T.; Gao, F. Enhanced IMC design of load disturbance rejection for integrating and unstable processes with slow dynamics. ISA Transactions 2011, 50(2), 239-248.

[12] Jin, Q. B.; Liu, Q. Analytical IMC-PID design in terms of performance/robustness tradeoff for integrating processes: From 2-Dof to 1-Dof. Journal of Process Control 2014, 24(3), 
22-32.

[13] Mamat, R. A new tuning method for two-degree-of-freedom internal model control under parametric uncertainty. Chinese Journal of Chemical Engineering 2013, 21(9), 1030-1037.

[14] Tan, W. Unified tuning of PID load frequency controller for power systems via IMC. IEEE Transactions on Power Systems 2010, 25(1), 341-350.

[15] Tan, W. Analysis and design of a double two-degree-of-freedom control scheme. ISA Transactions 2010, 49(3), 311-317.

[16] Hamed, B.; Issa, W. A modified internal model control for unstable-time delayed system. International Journal of Engineering and Advanced Technology 2011, 1(1), 56-62.

[17] Smith, O. J. M. Closer control of loops with dead time. Chemical Engineering Progress 1957, 53(5), 217-219.

[18] Guzmán, J. L.; Garcia, P.; Hägglund, T. et al. Interactive tool for analysis of time-delay systems with dead-time compensators. Control Engineering Practice 2008, 16(7), 824-835.

[19] Normey-Rico, J. E.; Camacho, E. F. Dead-time compensators: A survey. Control Engineering Practice 2008, 16(4), 407-428.

[20] García, P.; Albertos, P.; Hägglund, T. Control of unstable non-minimum-phase delayed systems. Journal of Process Control 2006, 16(10), 1099-1111.

[21] García, P.; Albertos, P. A new dead-time compensator to control stable and integrating processes with long dead-time. Automatica 2008, 44(4), 1062-1071.

[22] García, P.; Santos, T.; Normey-Rico, J. E. et al. Smith predictor-based control schemes for dead-time unstable cascade processes. Industrial \& Engineering Chemistry Research 2010, 49(22), 11471-11481.

[23] Torrico, B. C.; Normey-Rico, J. E. 2DOF discrete dead-time compensators for stable and integrative processes with dead-time. Journal of Process Control 2005, 15(3), 341-352.

[24] Normey-Rico, J. E.; Camacho, E. F. Unified approach for robust dead-time compensator design. Journal of Process Control 2009, 19(1), 38-47.

[25] Ajmeri, M.; Ali, A. Simple tuning rules for integrating processes with large time delay. Asian Journal of Control 2015. (DOI:10.1002/asjc.1119). 
[26] Mataušek, M. R.; Ribić, A. I. Control of stable, integrating and unstable processes by the modified Smith Predictor. Journal of Process Control 2012, 22(1), 338-343.

[27] Rao, A. S.; Chidambaram, M. Analytical design of modified Smith predictor in a two-degrees-of-freedom control scheme for second order unstable processes with time delay. ISA Transactions 2008, 47(4),407-419.

[28] Rao, A. S.; Rao, V. S. R., Chidambaram M. Simple analytical design of modified Smith predictor with improved performance for unstable first-order plus time delay (FOPTD) processes. Industrial \& Engineering Chemistry Research 2007, 46(13), 4561-4571.

[29] Ribić, A. I.; Mataušek. M. R. A dead-time compensating PID controller structure and robust tuning. Journal of Process Control 2012, 22(7), 1340-1349

[30] Flesch, R. C. C.; Torrico, B. C.; Normey-Rico, J. E.; Cavalcante, M.U. Unified approach for minimal output dead time compensation in MIMO processes. Journal of Process Control 2011, 21 (7), 1080-1091.

[31] Cong, E. -D.; Hu, M. -H.; Tu, S. -T. et al. A novel double loop control model design for chemical unstable processes. ISA Transactions 2014, 53, 497-507.

[32] Normey-Rico, J .E.; Sartori, R.; Veronesi, M. and Visiol, A. An automatic tuning methodology for a unified dead-time compensator. Control Engineering Practice 2014, 27, $11-22$

[33] Zhang, G.; Zhang, X.; Guan, W. Stability analysis and design of integrating unstable delay processes using the mirror-mapping technique. Journal of Process Control 2014, 24(7), 1038-1045.

[34] Zhao, S.; Gao, Z. Modified active disturbance rejection control for time-delay systems. ISA Transactions 2014, 53(4), 882-888.

[35] Albertos, P.; García, P. Robust control design for long time-delay systems, Journal of Process Control 2009, 19(10), 1640-1648.

[36] García, P.; Albertos, P. Robust tuning of a generalized predictor-based controller for integrating and unstable systems with long time-delay. Journal of Process Control 2013, 23(8), 1205-1216. 
[37] Gu, D.; Ou, L.; Wang, P. Relay feedback autotuning method for integrating processes with inverse response and time delay. Industrial \& Engineering Chemistry Research 2006, 45(9), 3119-3132.

[38] Luyben, W. L. Identification and tuning of integrating processes with deadtime and inverse response. Industrial \& Engineering Chemistry Research 2003, 42(13), 3030-3035. 


\section{Table and Figure Captions}

Table 1 Summary of the controller design formulae

Table 2 IAE for set-point tracking by different methods

Table 3 IAE for load disturbance rejection by different methods

Figure 1 The predictor-based control structure

Figure 2 Ideal delay-free control loop

Figure 3 The predictor-based control structure with process uncertainty

Figure 4 The $M-\Delta$ structure for robust stability analysis

Figure 5 Control results for Example 1

Figure 6 Perturbed system responses of Example 1

Figure 7 Illustration on a trade-off between control performance and robust stability

Figure 8 Numerical relationship between the disturbance response peak and $\lambda_{\mathrm{c}}$ for Example 1

Figure 9 Numerical results regarding different values of the time delay for Example 1

Figure 10 Control results for Example 2

Figure 11 Perturbed system responses of Example 2

Figure 12 Control results for Example 3

Figure 13 Perturbed system responses of Example 3

Figure 14 Control results for Example 4

Figure 15 Perturbed system responses of Example 4

Figure 16 Numerical relationship between the disturbance response peak and $\lambda_{\mathrm{c}}$ regarding the time delay for Example 4

Table 1 Summary of the controller design formulae

\begin{tabular}{lll}
\hline Process model & \multicolumn{1}{c}{$K$} & \multicolumn{1}{c}{$K_{\mathrm{f}}$} \\
\hline \multirow{3}{*}{ eq.(12) } & eq.(26) for $0<z_{0}<1$ & eqs.(47)-(51), \\
& eq.(28) for $-1<z_{0}<0$ & eqs.(23)-(25) for $\left|z_{0}\right|<1$ \\
& eq.(39) for $\left|z_{0}\right|>1$ & eqs.(36)-(38) for $\left|z_{0}\right|>1$ \\
\hline eq.(13) & eq.(33) for $0<z_{0}<1$ & eqs.(47)-(51), \\
& eq.(34) for $-1<z_{0}<0$ & eqs.(23), (29), (30) for $\left|z_{0}\right|<1$ \\
& eqs.(43)-(44) for $\left|z_{0}\right|>1$ & eqs.(36), (40), (41) for $\left|z_{0}\right|>1$
\end{tabular}

(Note: A higher-order filter $\left(n_{\mathrm{f}} \geq 2\right)$ in (49) may be used to design $K_{\mathrm{f}}$ ) 

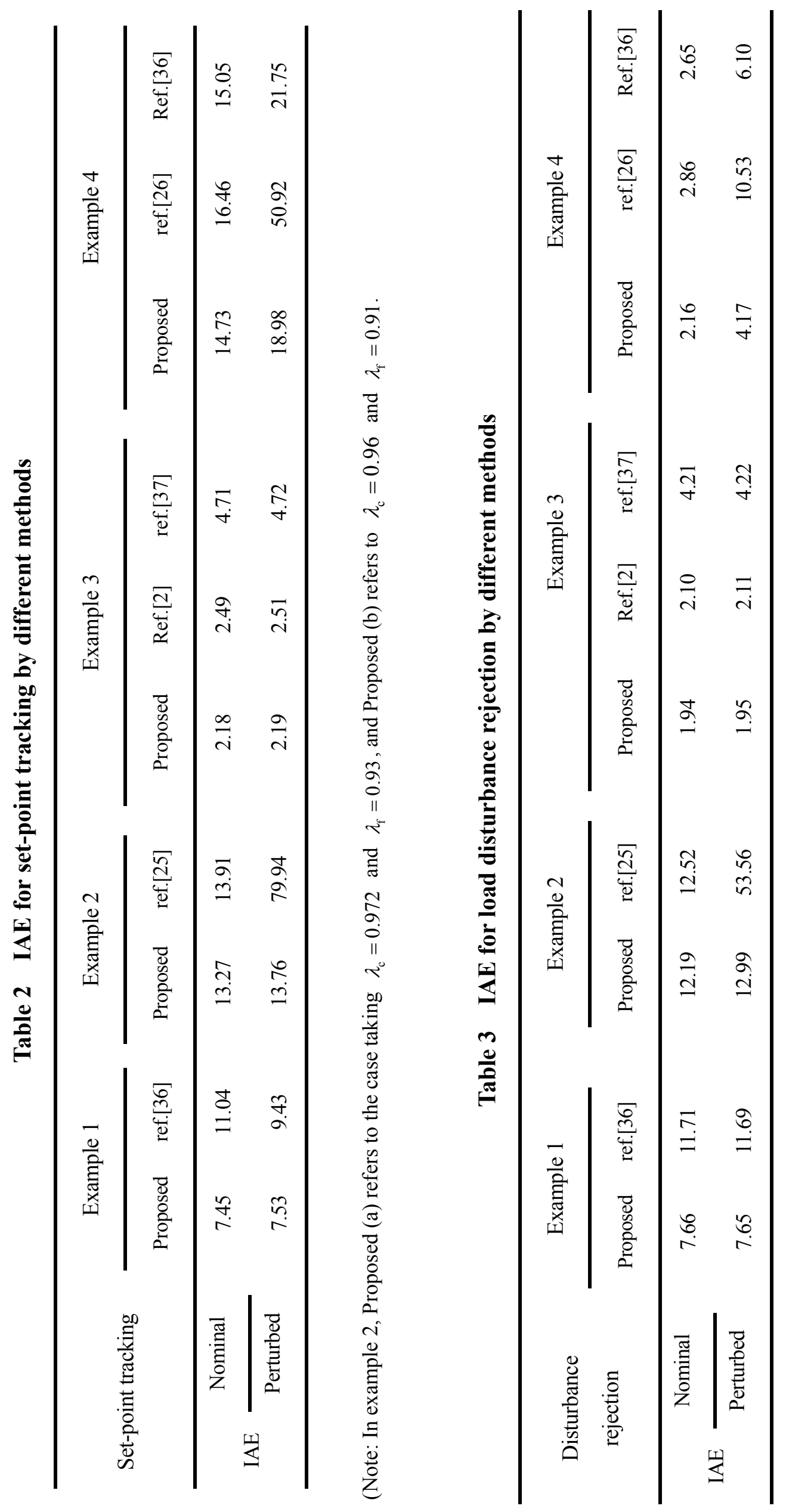


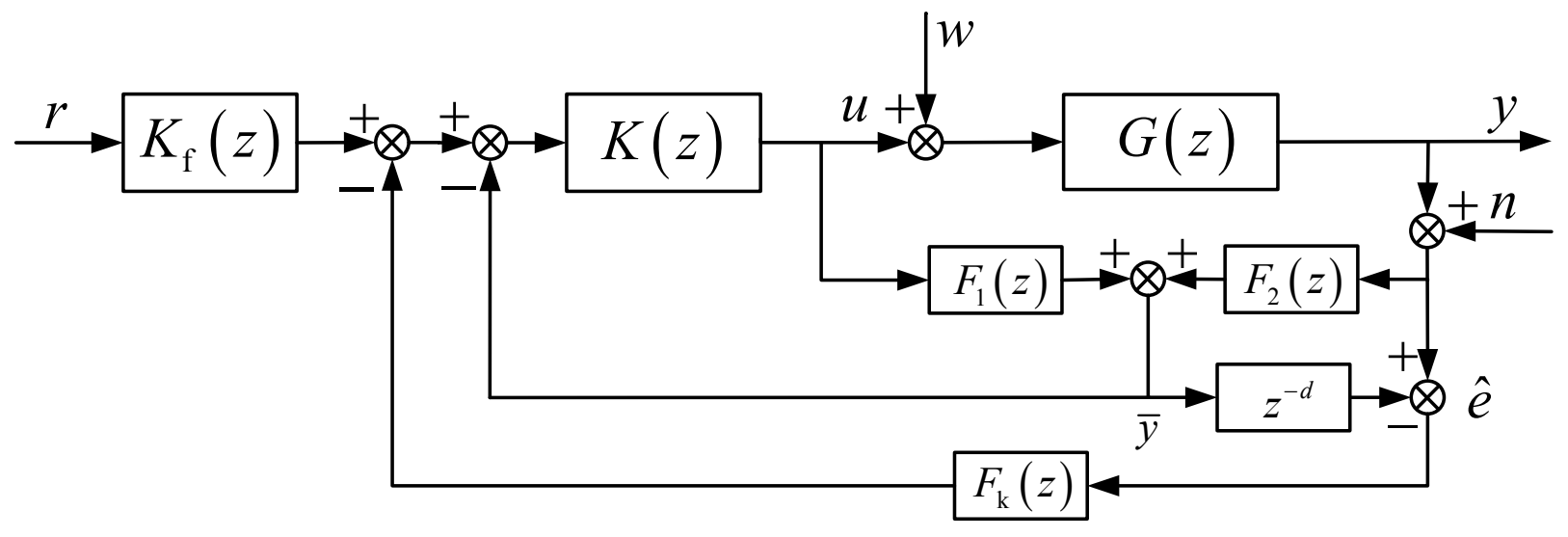

Figure 1 The predictor-based control structure

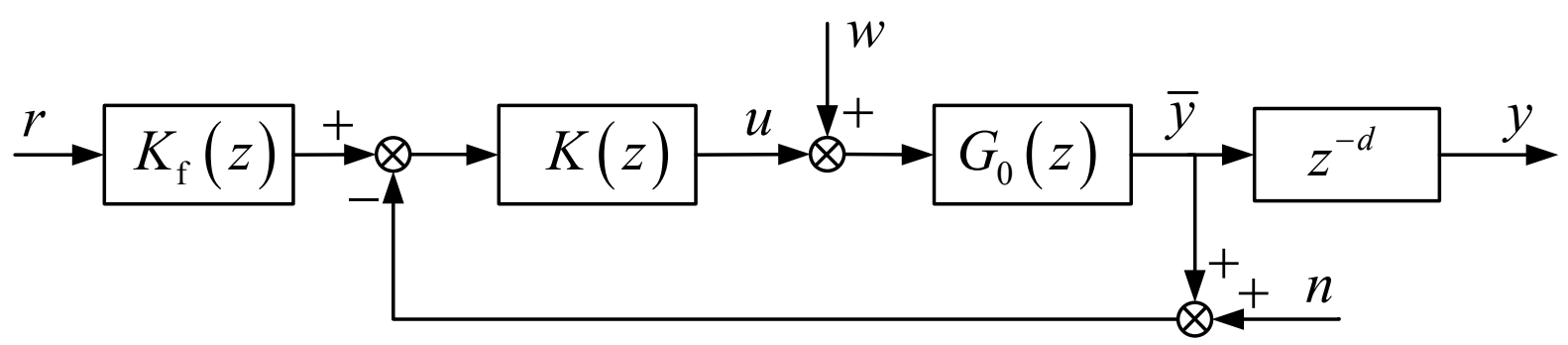

Figure 2 Ideal delay-free control loop

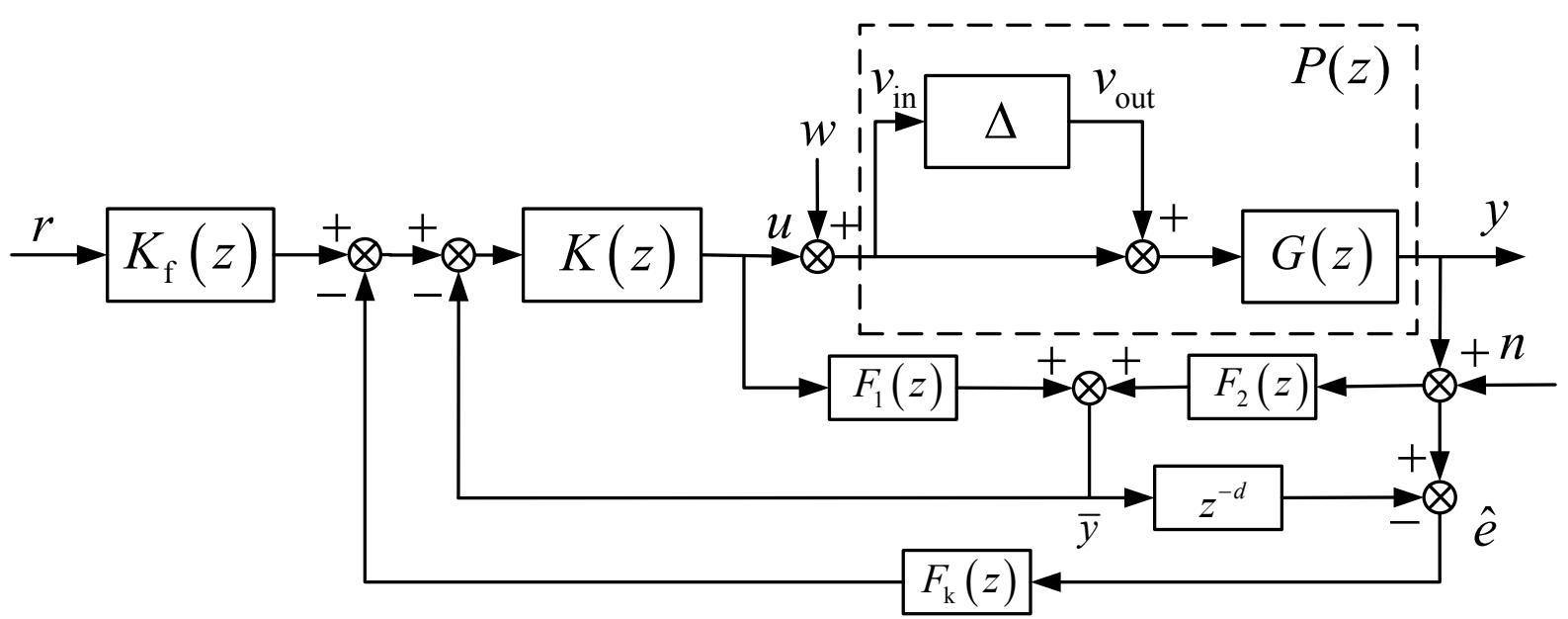

Figure 3 The predictor-based control structure with uncertainty 


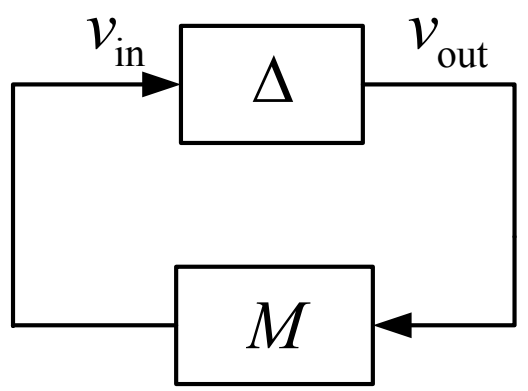

Figure 4 The $M-\Delta$ structure for robust stability analysis

(a)

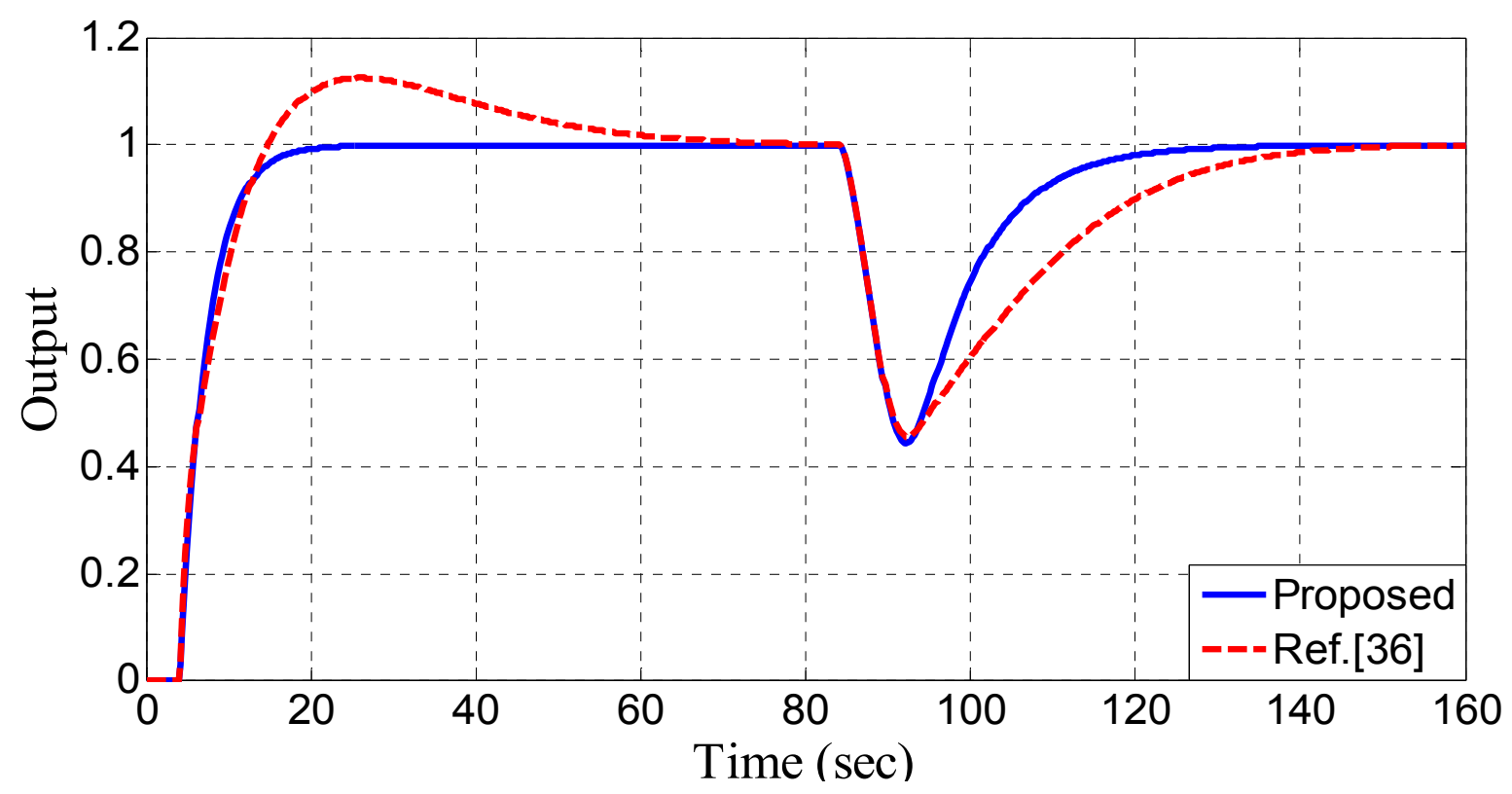

(b)

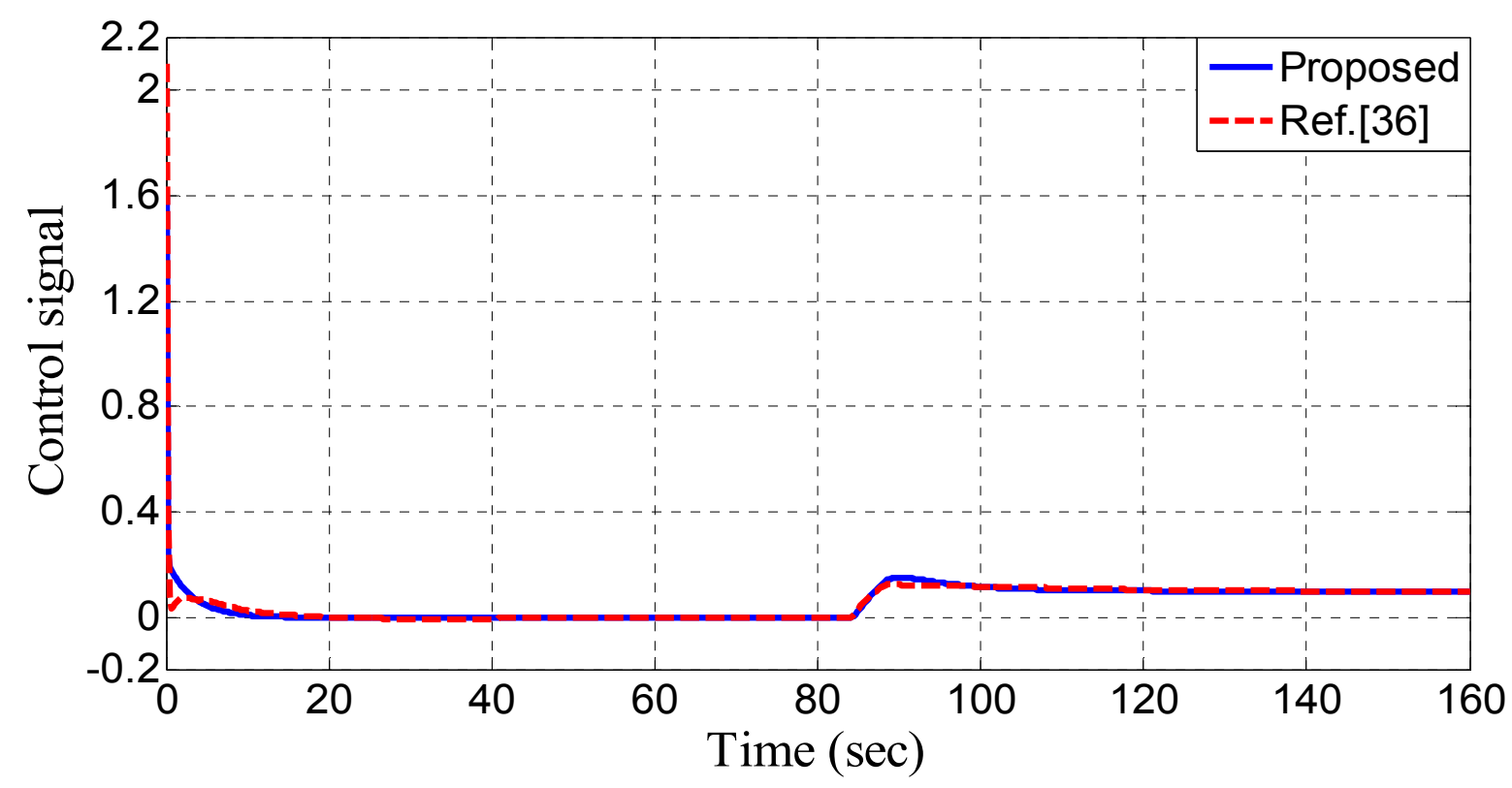

Figure 5 Control results for Example 1 
(a)

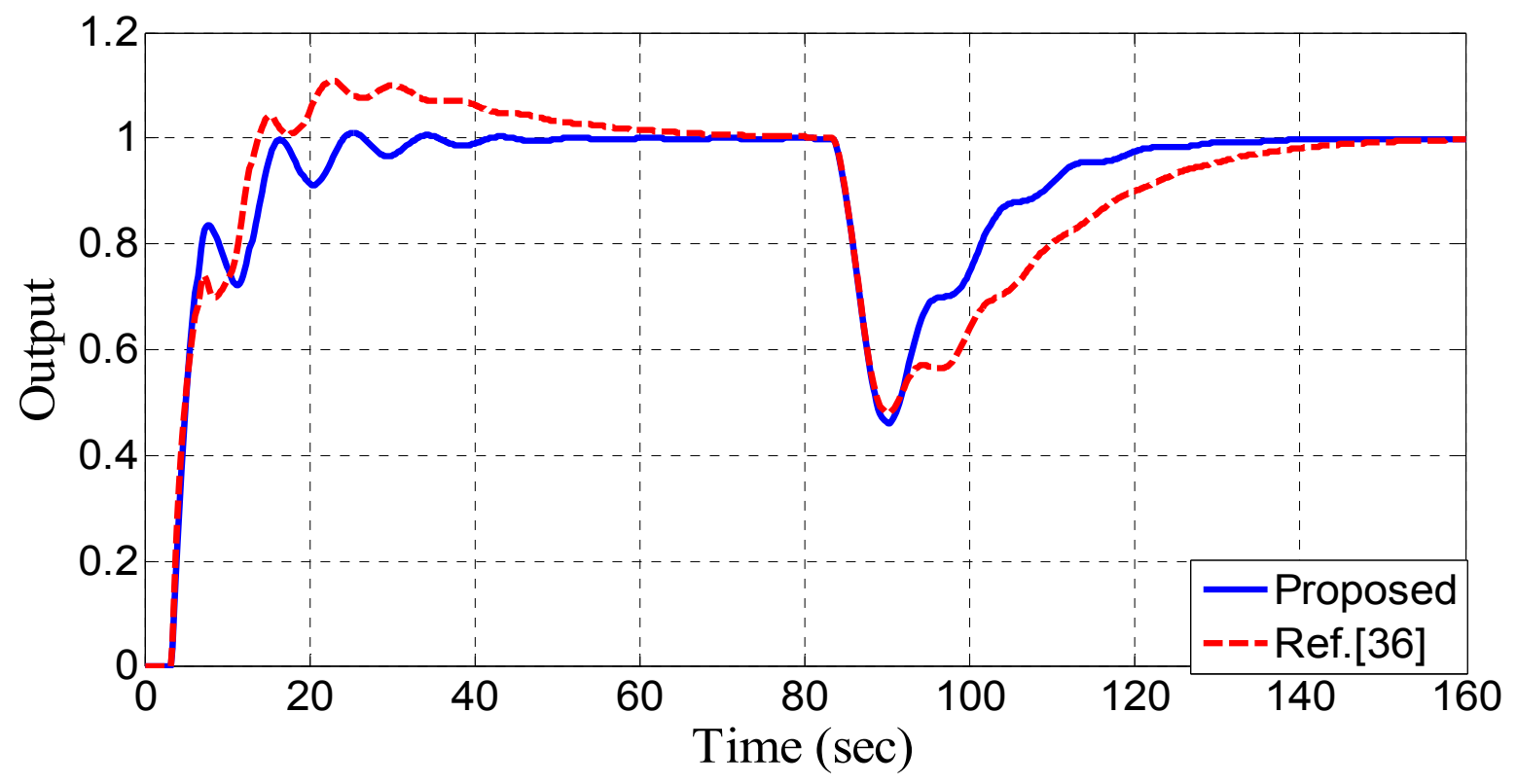

(b)

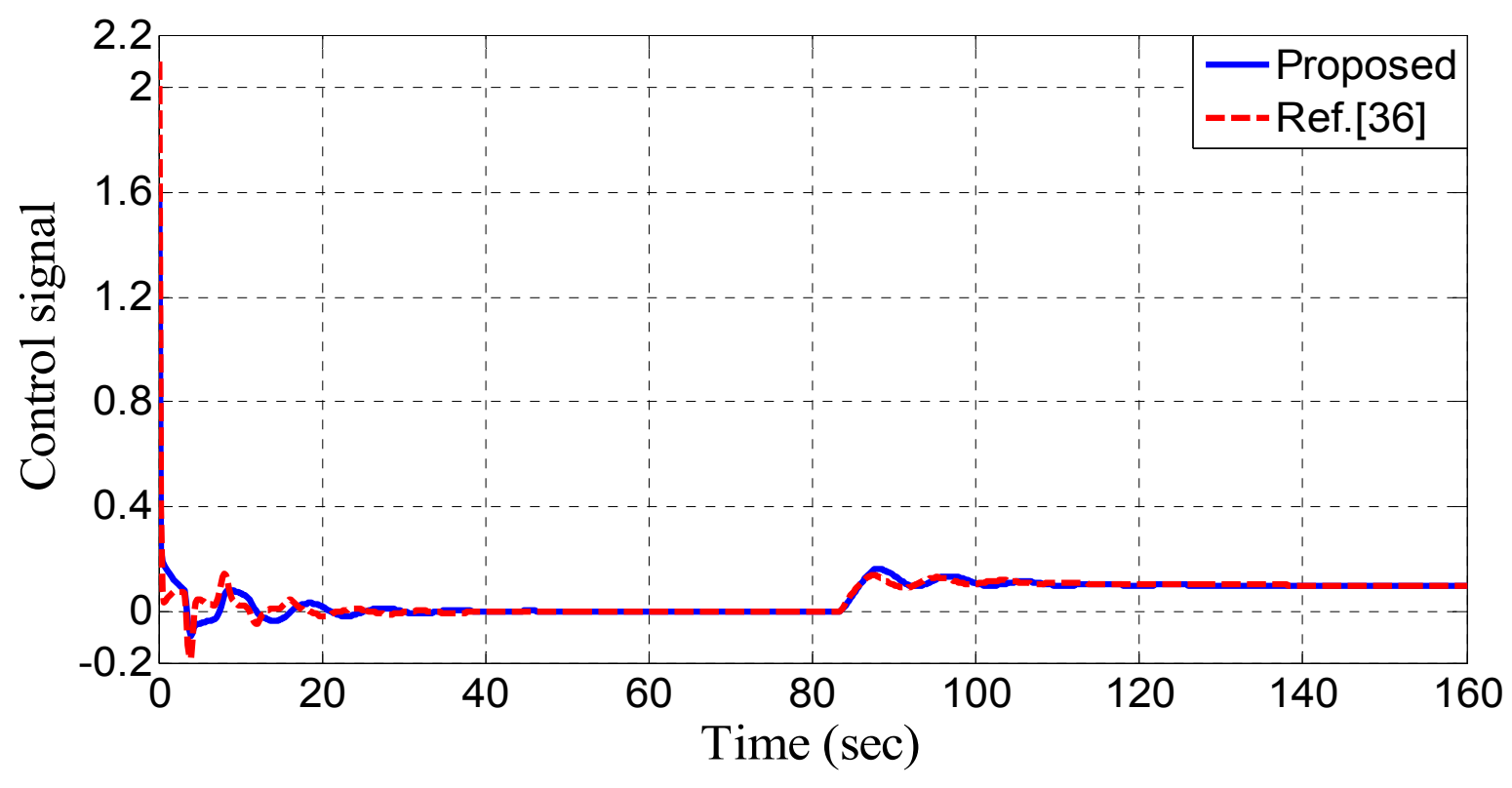

Figure 6 Perturbed system responses of Example 1 


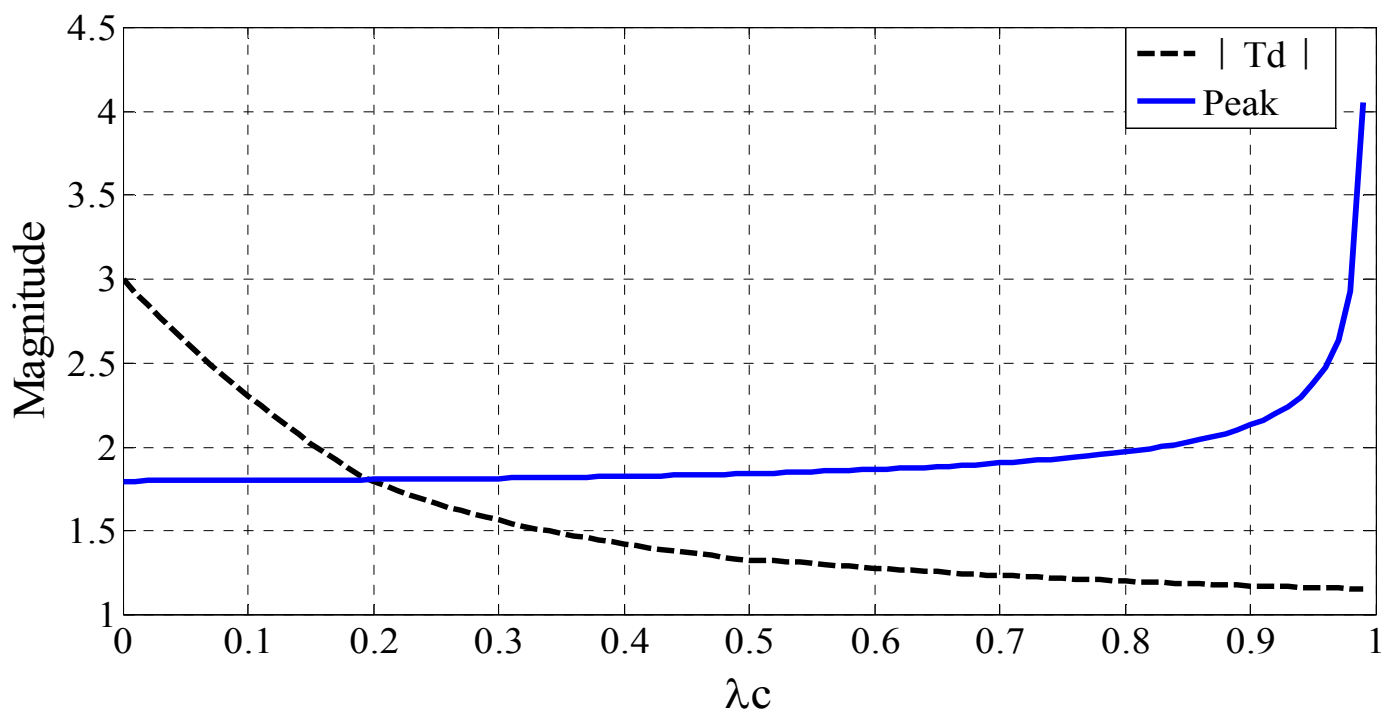

Figure 7 Illustration on a trade-off between control performance and robust stability

(a)

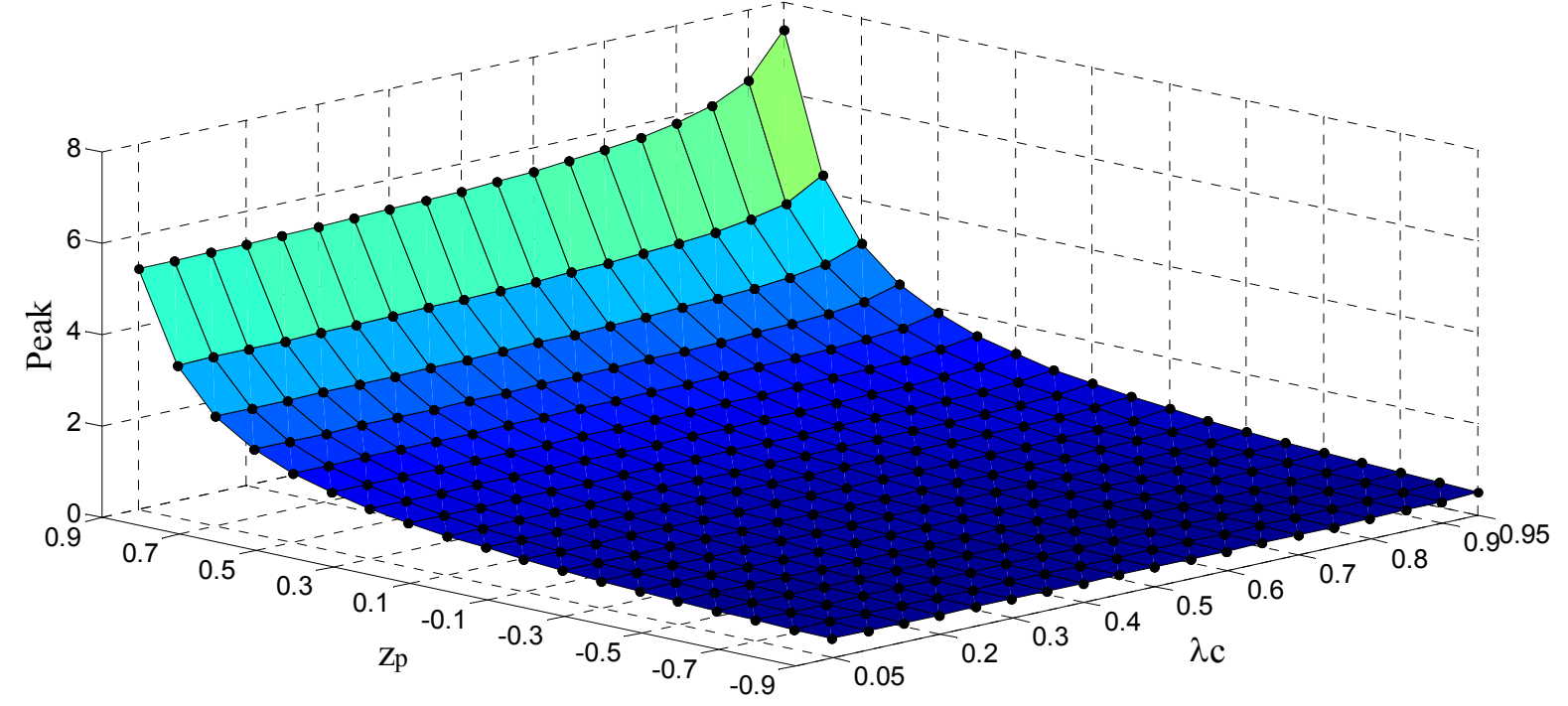

(b)

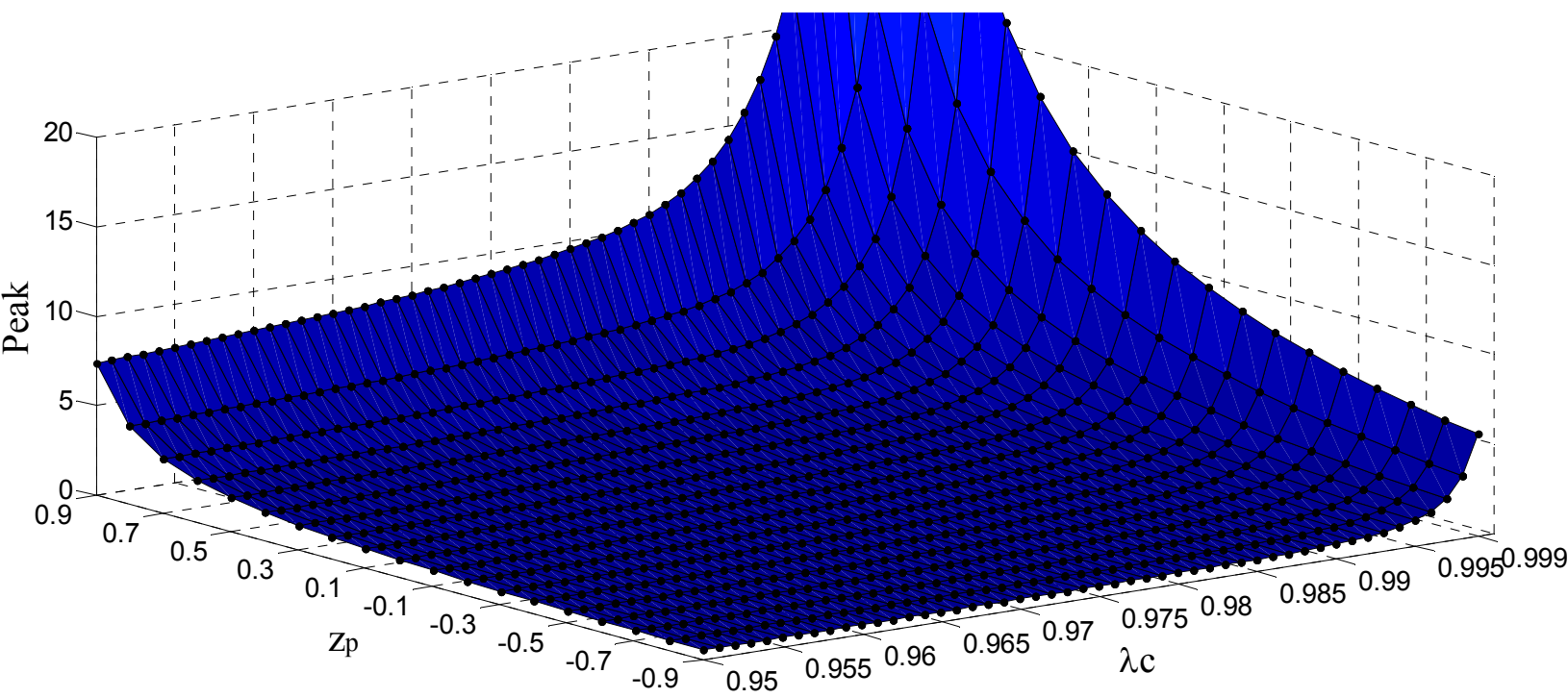

Figure 8 Numerical relationship between the disturbance response peak and $\lambda_{\mathrm{c}}$ for

\section{Example 1}




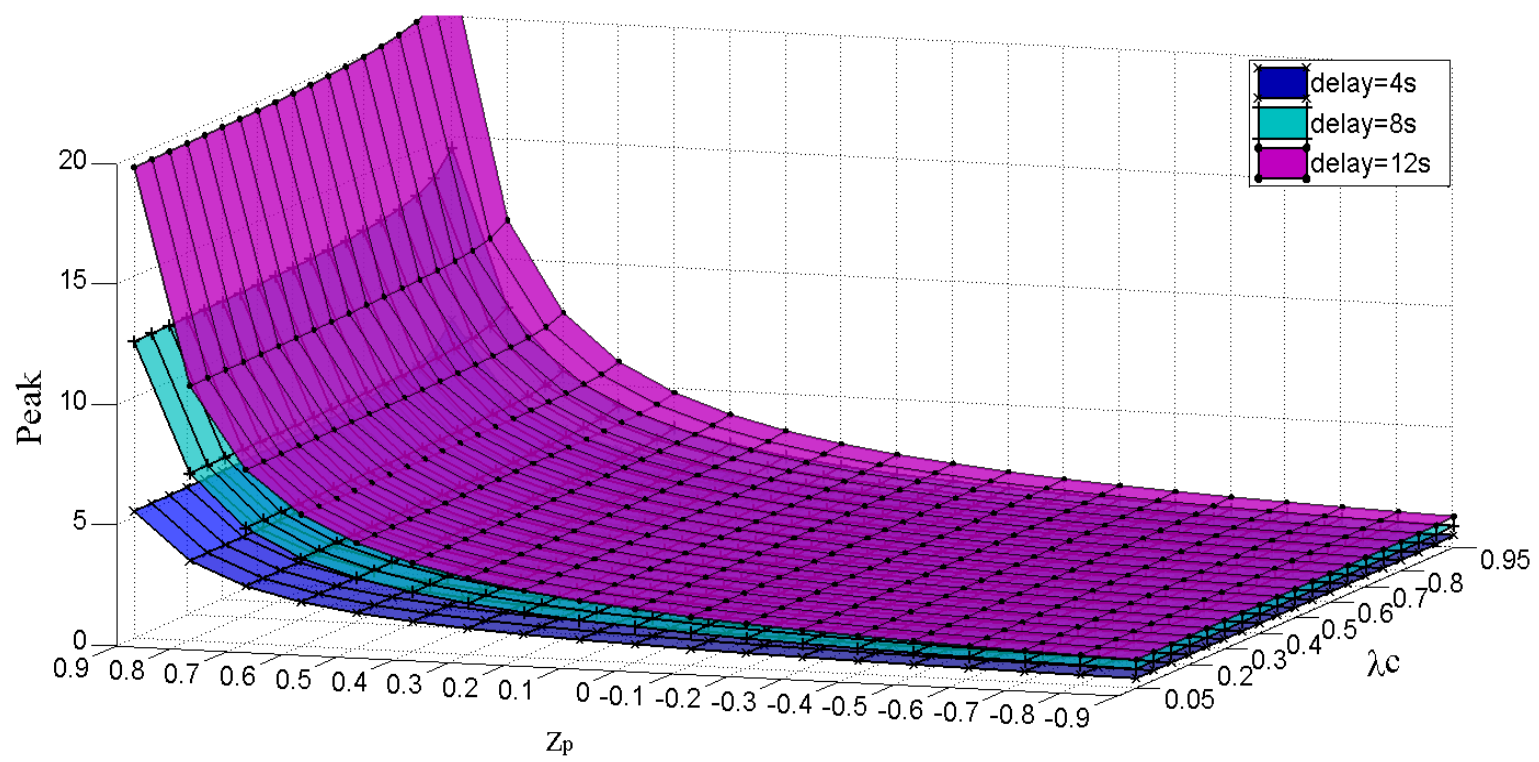

Figure 9 Numerical results regarding different values of the time delay for Example 1 
(a)

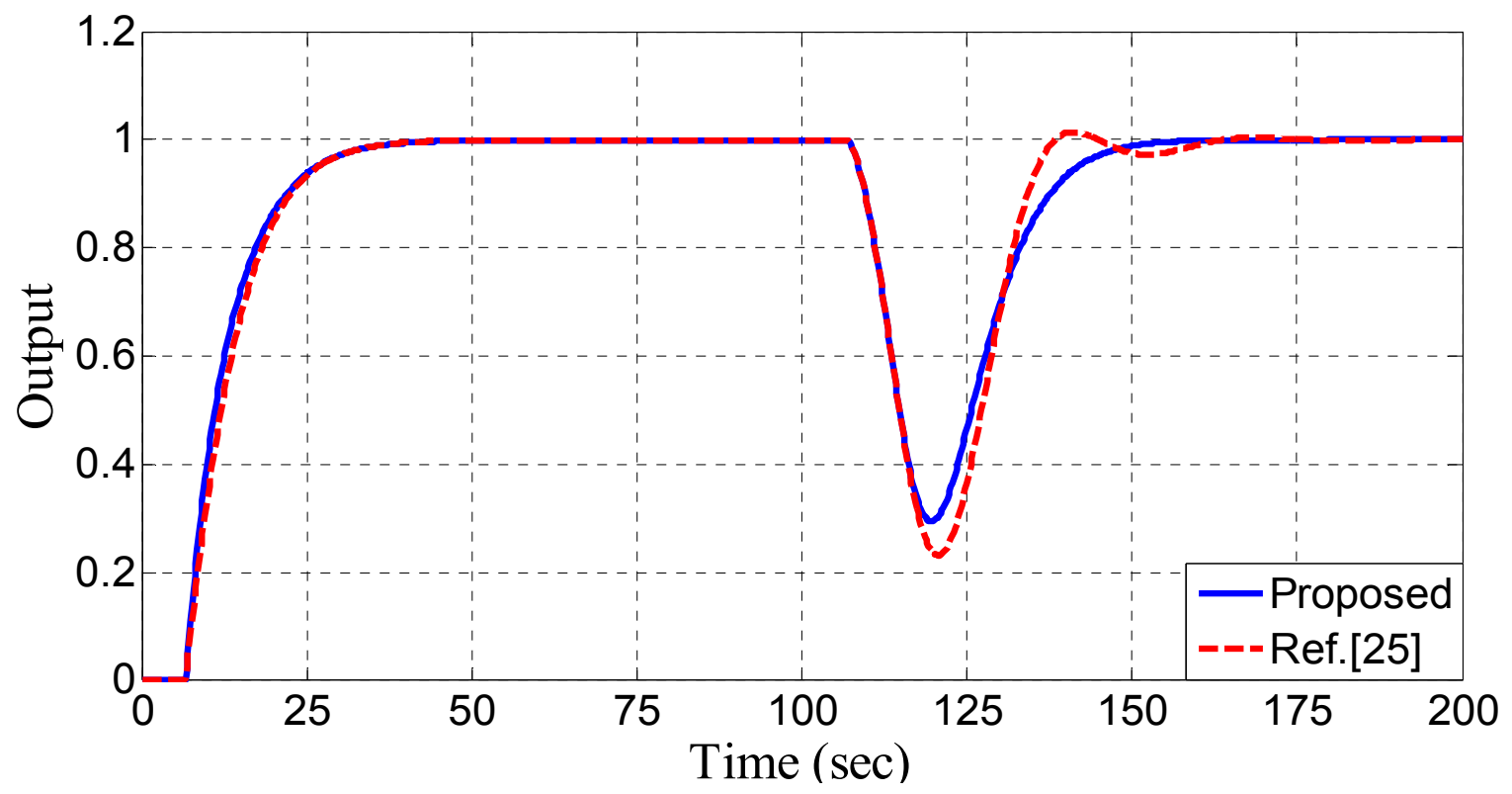

(b)

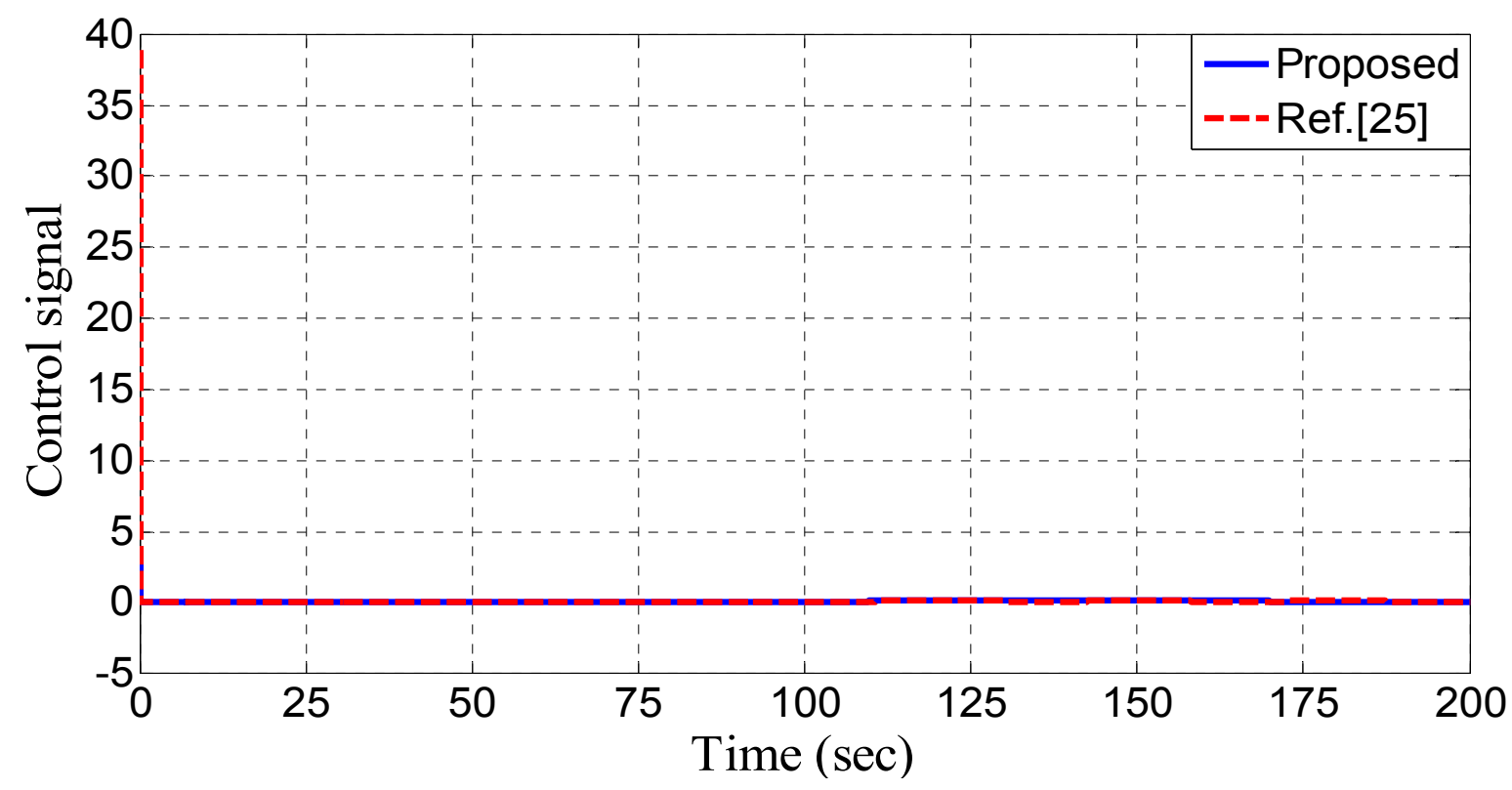

Figure 10 Control results for Example 2 
(a)

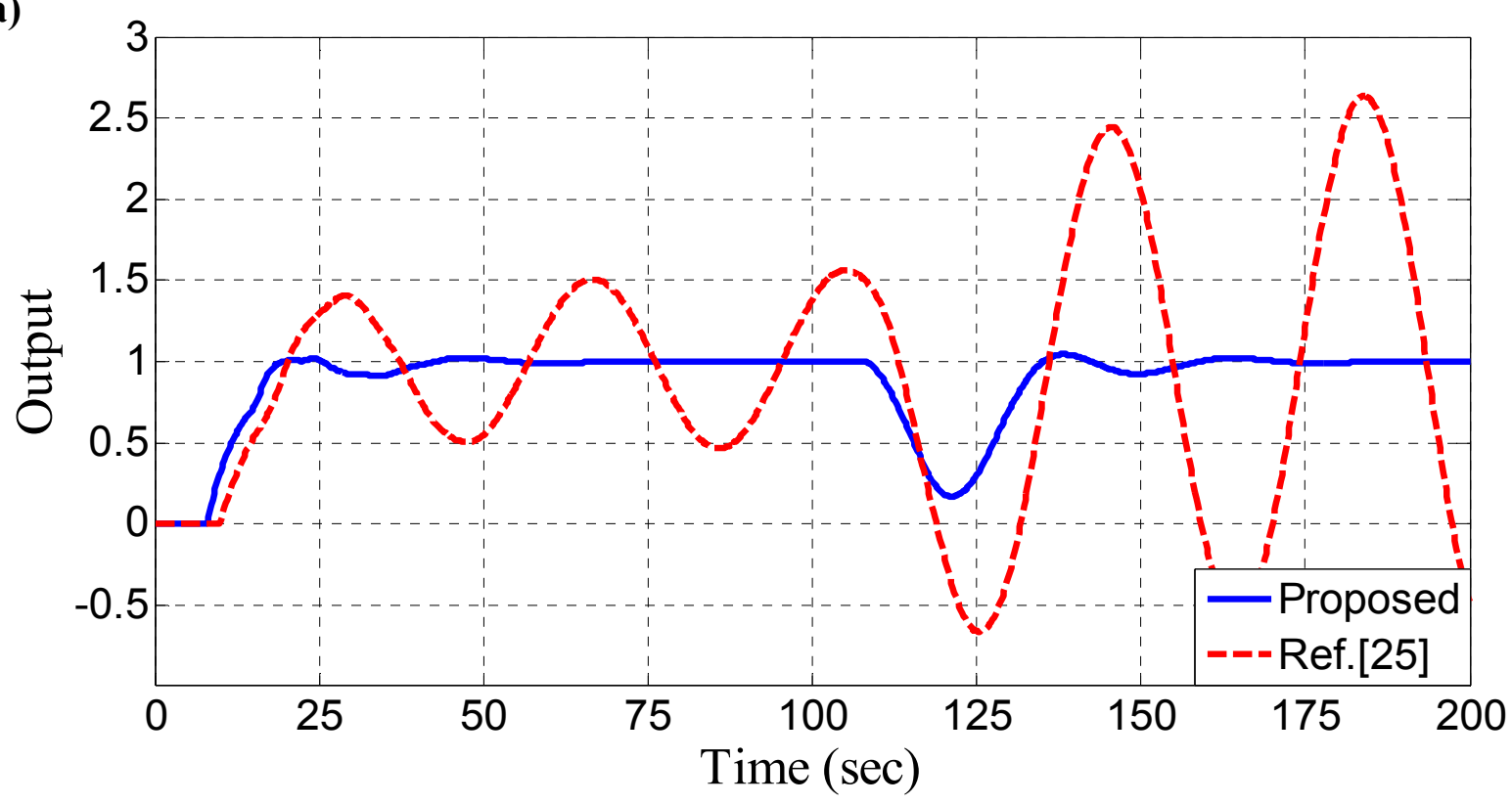

(b)

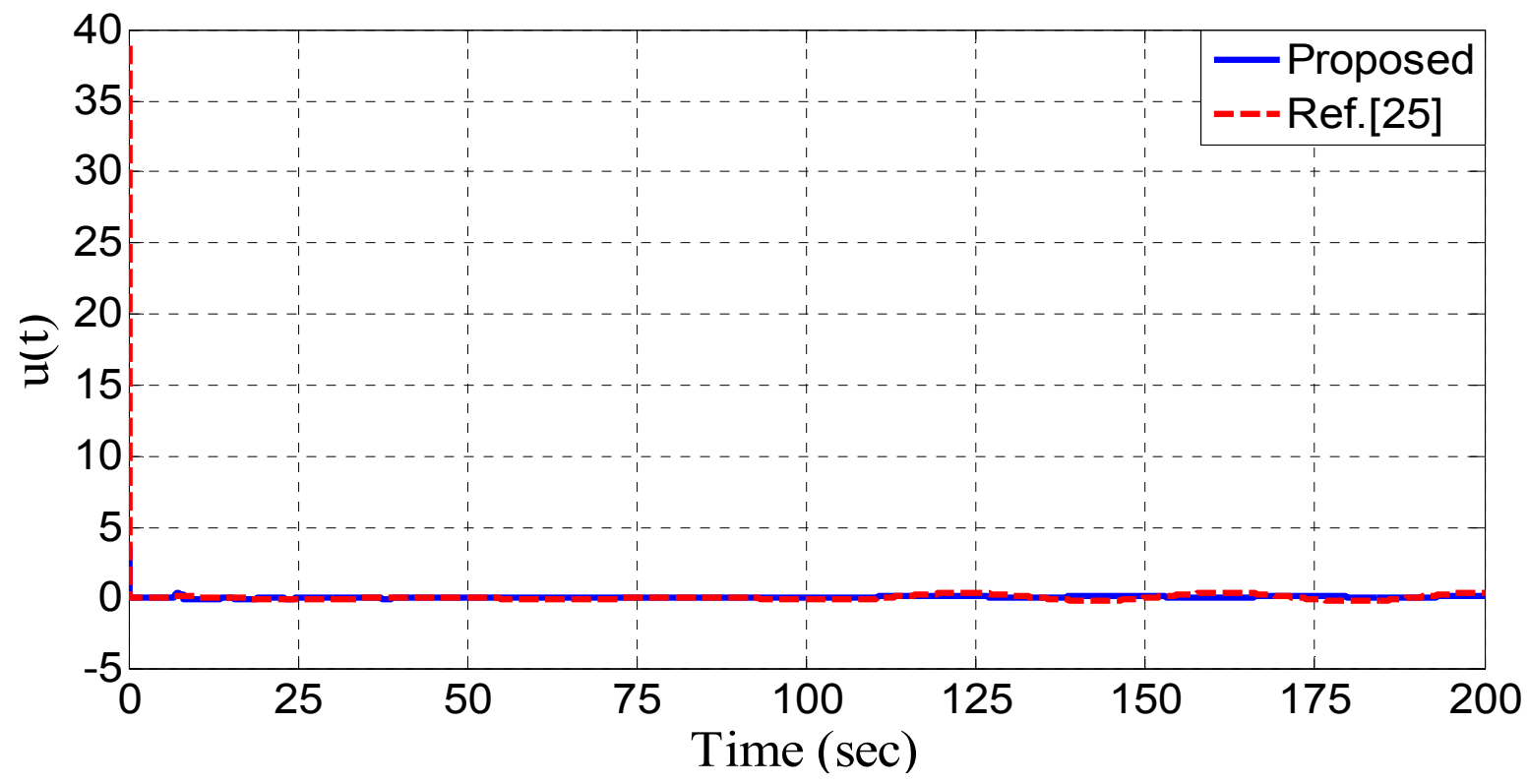

Figure 11 Perturbed system responses of Example 2 
(a)

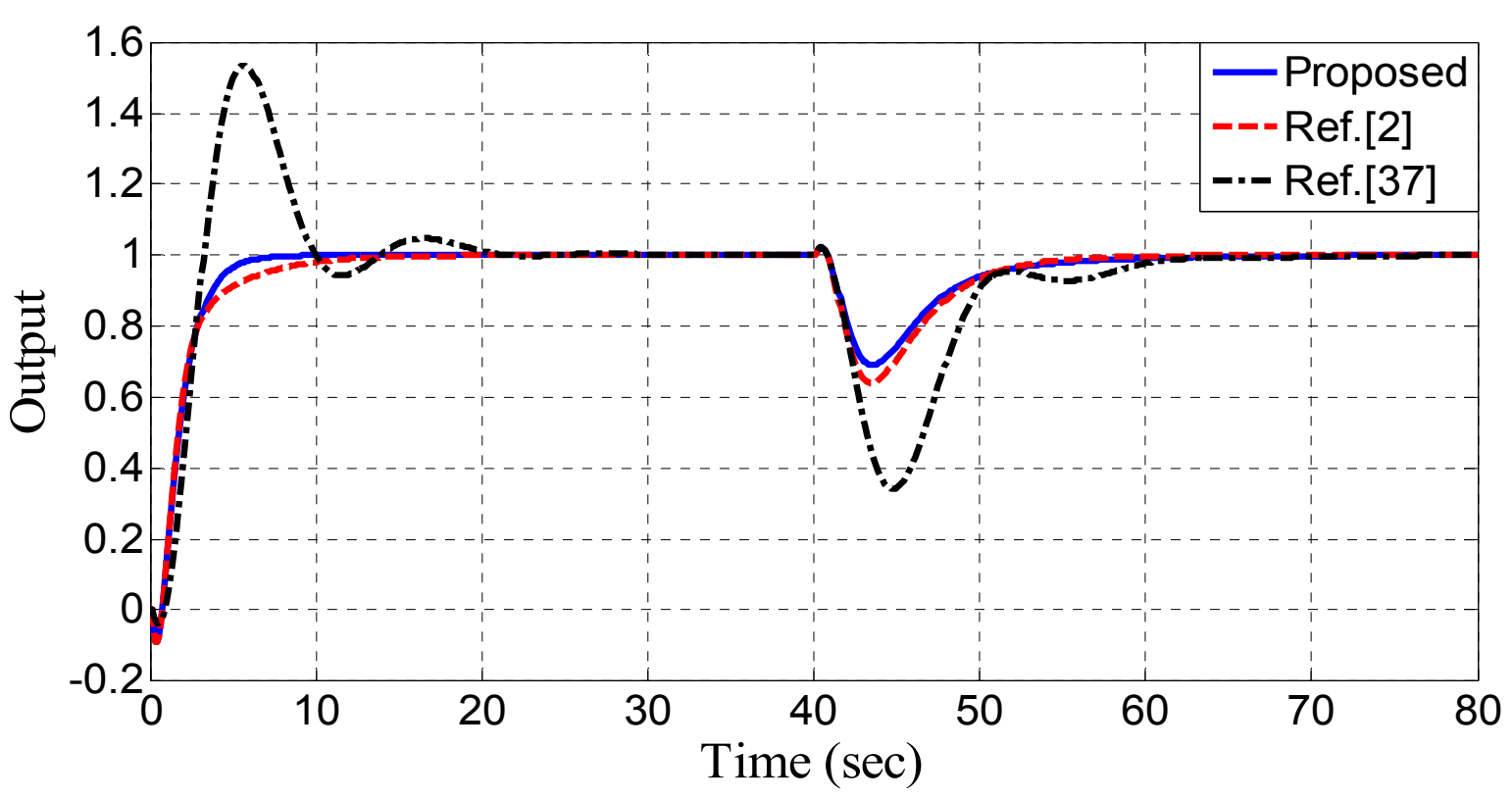

(b)

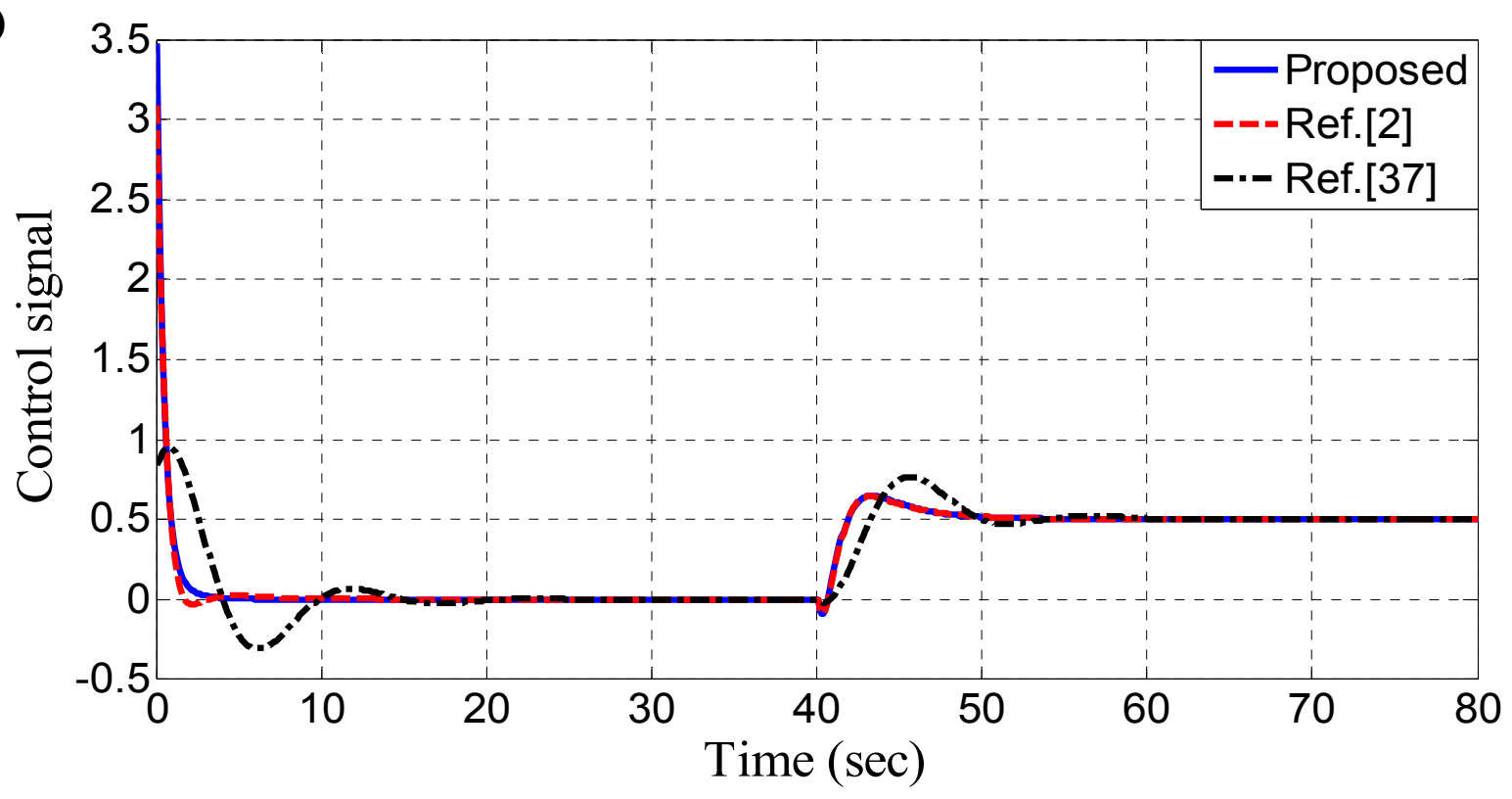

Figure 12 Control results for Example 3 
(a)

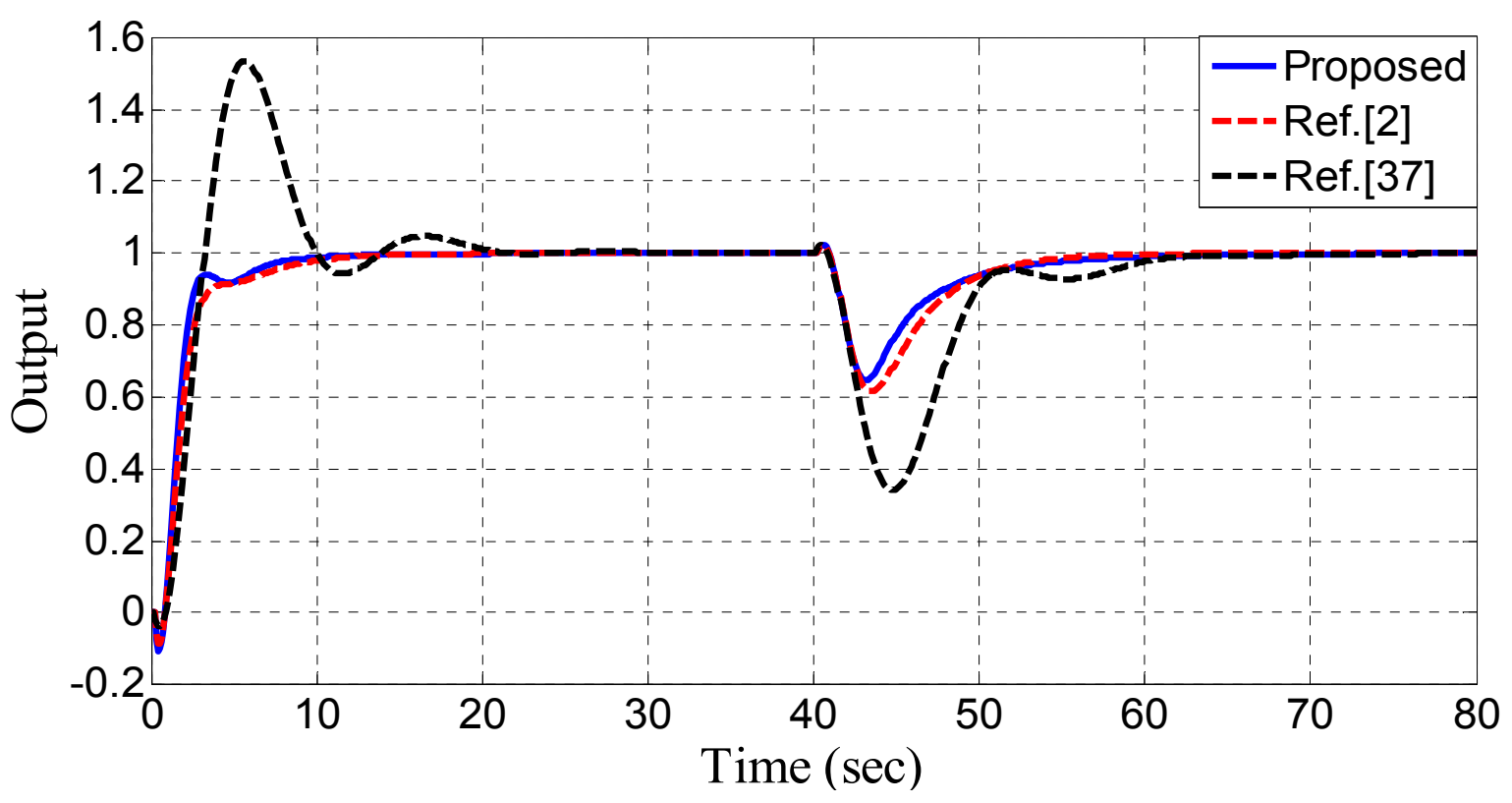

(b)

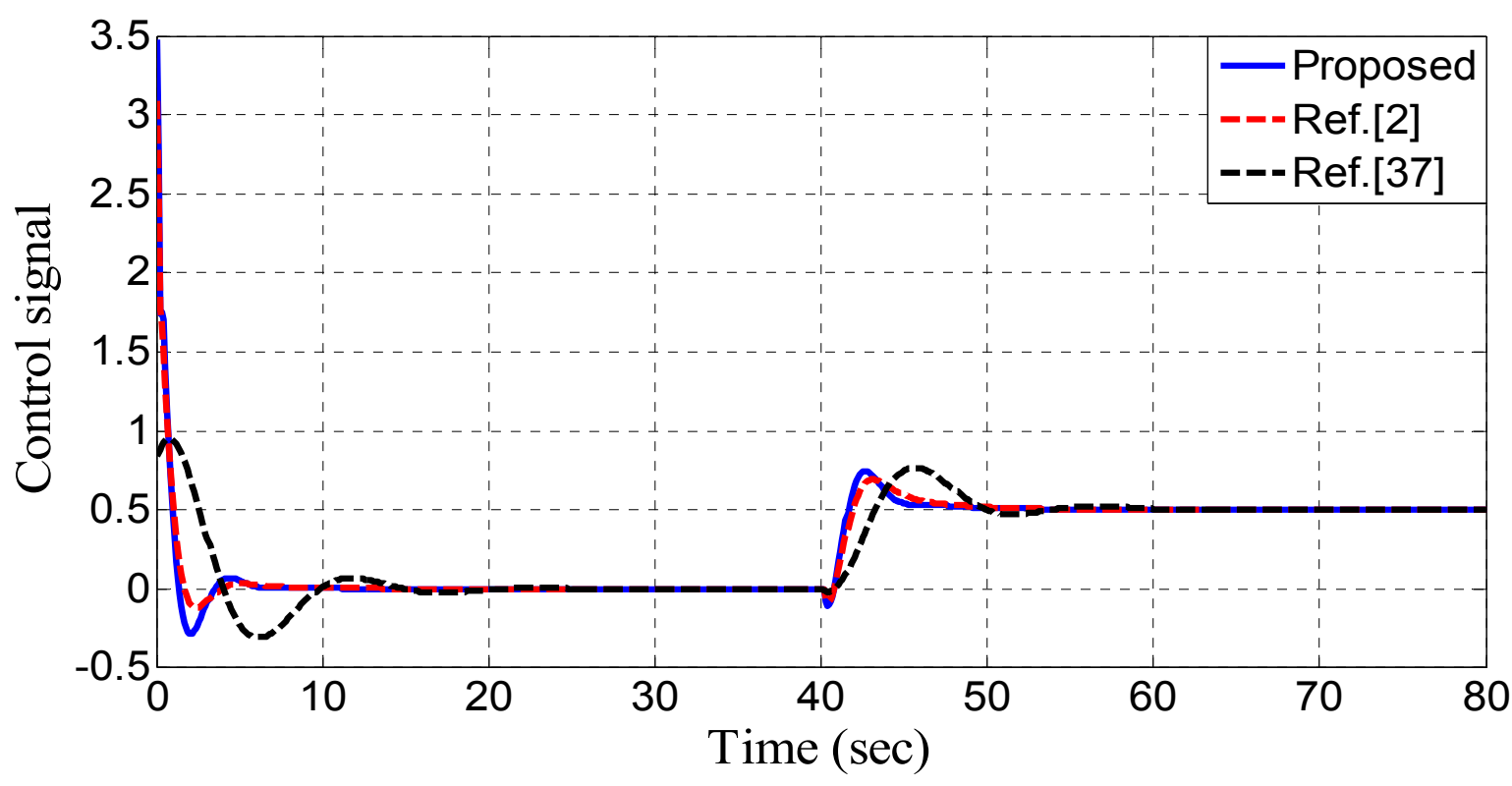

Figure 13 Perturbed system responses of Example 3 
(a)

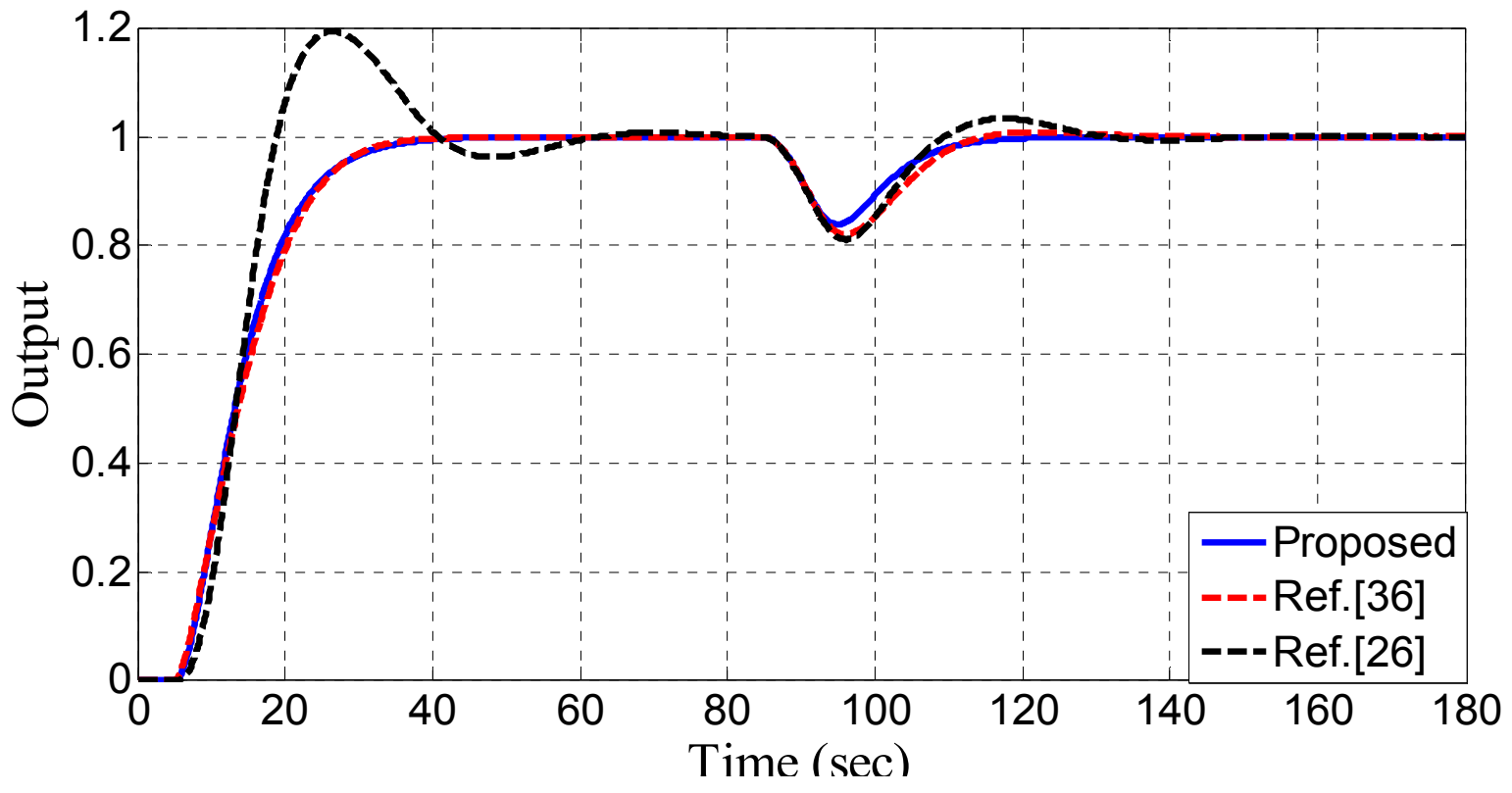

(b)

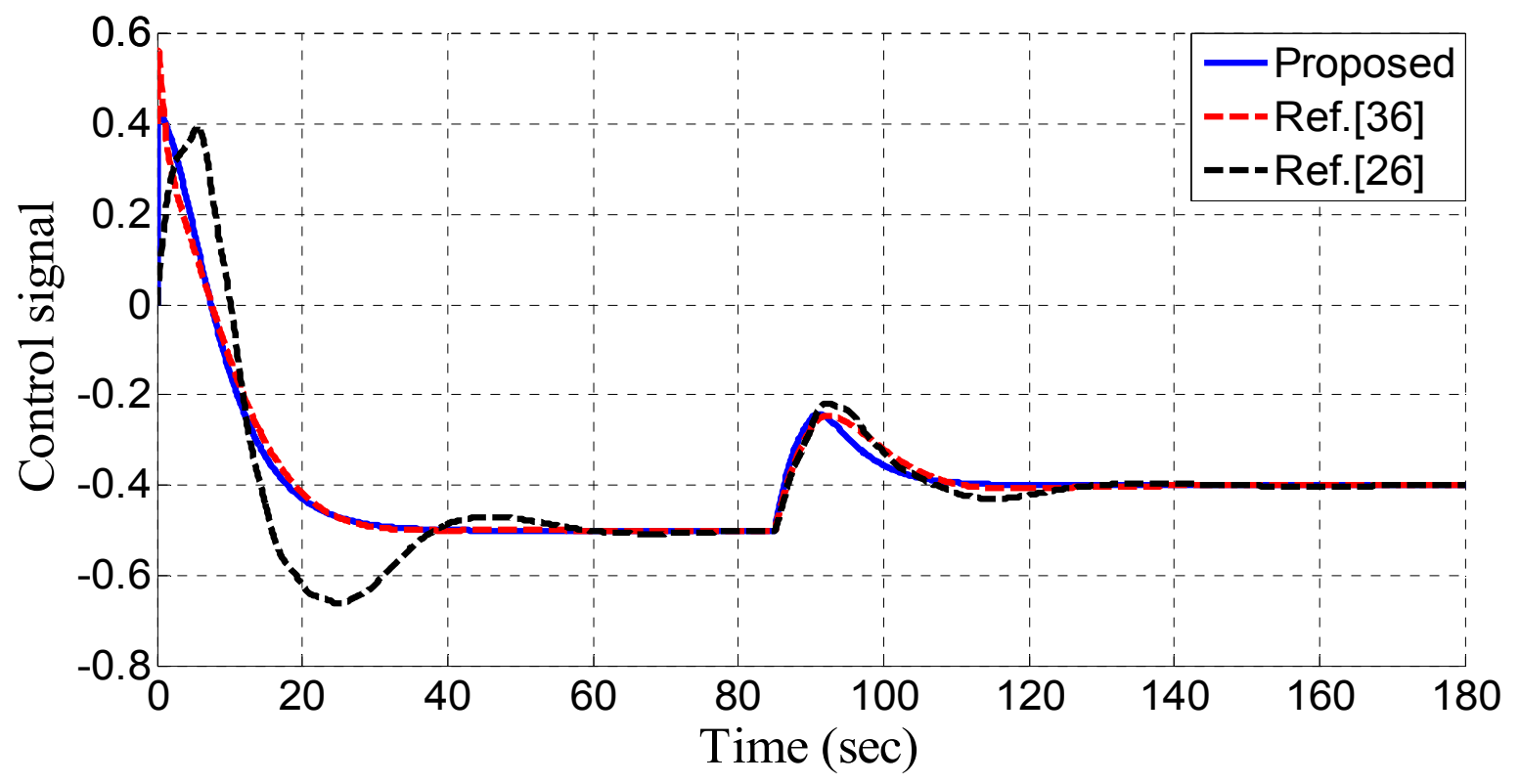

Figure 14 Control results for Example 4 

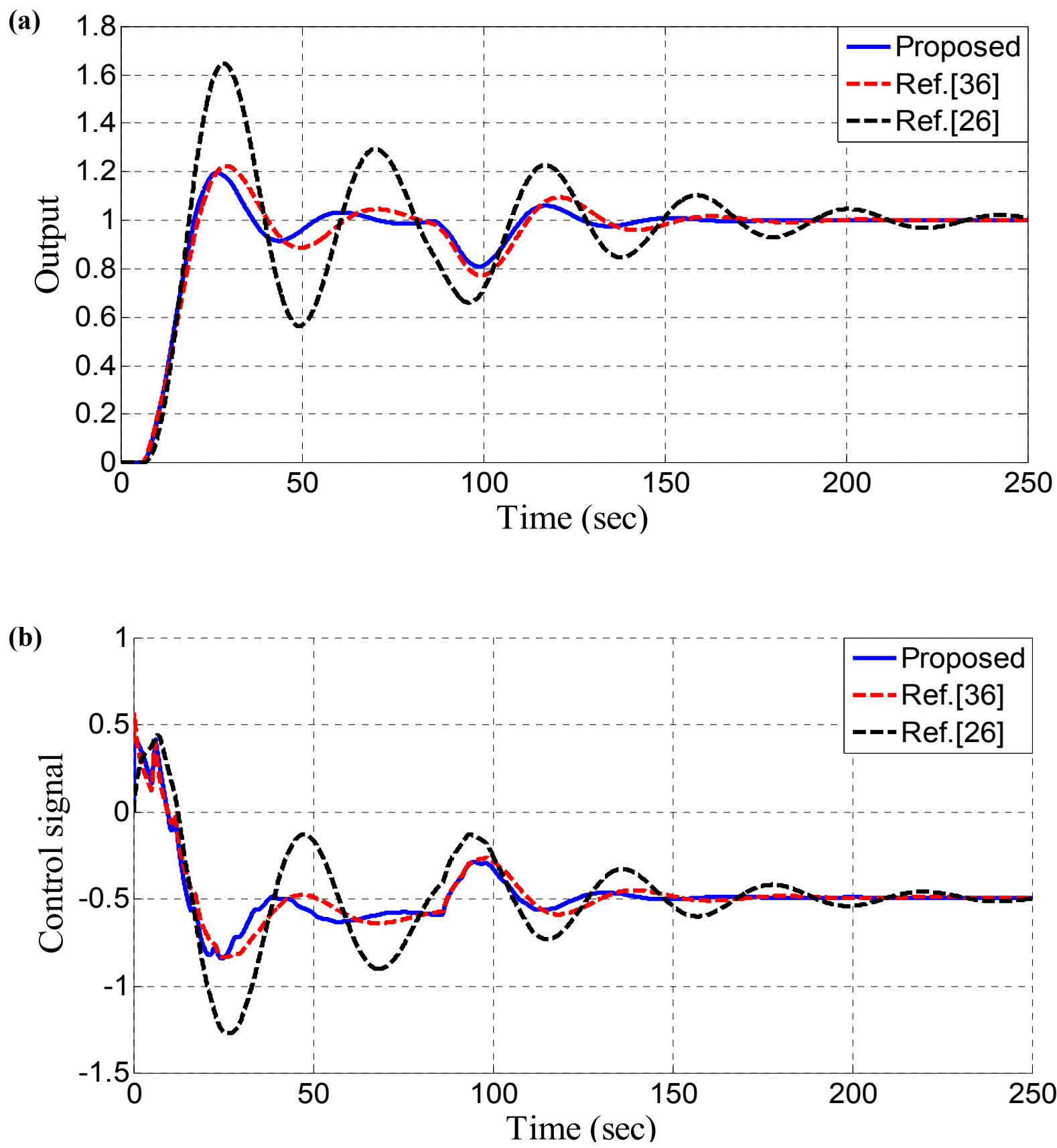

Figure 15 Perturbed system responses of Example 4 


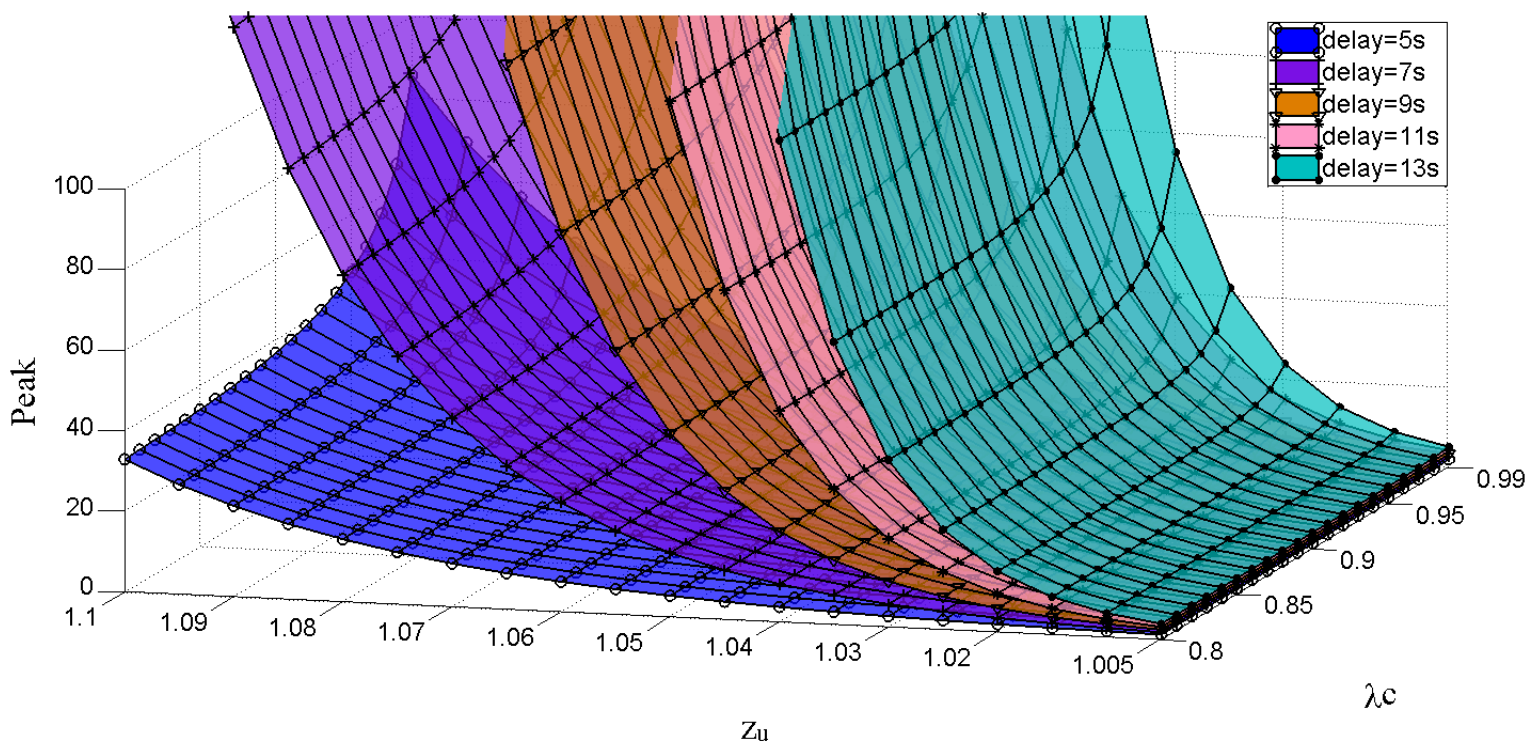

Figure 16 Numerical relationship between the disturbance response peak and $\lambda_{\mathrm{c}}$ regarding the time delay for Example 4 